\title{
Accuracy Evaluation of Broadcast Ephemeris for BDS-2 and BDS-3
}

\author{
Hao Jun Jian ( $\nabla 1977817186 @ q q . c o m$ ) \\ Wuhan University https://orcid.org/0000-0001-9171-0881 \\ Yishi Wang \\ Wuhan University \\ Shoujian Zhang \\ Wuhan University
}

Full paper

Keywords: BDS-3, Broadcast ephemeris, SISRE, Clock error

Posted Date: April 5th, 2021

DOI: https://doi.org/10.21203/rs.3.rs-366742/v1

License: (c) (1) This work is licensed under a Creative Commons Attribution 4.0 International License.

Read Full License 
$3 \bowtie$ Haojun Jian

\section{1977817186@qq.com}

$5 \quad{ }^{1}$ School of Geodesy and Geomatics, Wuhan University, 129 Luoyu Road, Wuhan

6 430079, People's Republic of China

$$
\text { Haojun Jian }{ }^{1} \cdot \text { Yishi Wang }^{1} \cdot \text { Shoujian Zhang }{ }^{1}
$$

Abstract: The BDS-3 system was completed in July 2020 and began to provide services to users around the world. The inspection of its operation, especially the detailed evaluation of the orbit, clock error, TGD and other indicators, plays an important role in the subsequent positioning. This study conducts an investigation of

11 the satellite broadcast ephemeris of the BDS-2 and BDS-3. The difference between the 12 satellite orbit position calculated by the broadcast ephemeris and the position calculated 13 by the precise ephemeris is used for analysis. First, the ephemeris form January 2020 to February 2020 are investigated. The results show that the broadcast ephemeris accuracy of the BDS-2 MEO satellite is the highest, while the GEO satellite broadcast ephemeris accuracy is the lowest. And their three-dimensional orbit difference is $3 \mathrm{~m}$ and $7.5 \mathrm{~m}$, respectively. Second, the BDS-3 MEO satellite broadcast ephemeris accuracy is higher than the BDS-2, its three-dimensional orbit accuracy is about $0.39 \mathrm{~m}$, while its clock error is slightly smaller than the BDS-2. The result of ephemeris calculation is basically equivalent to the clock error of satellite-to-earth observation, which is related to the addition of the clock error of the inter-satellite link in the BDS3. Finally, the clock error of the BDS-3 MEO satellite with the H clock is basically the same as that of the MEO satellite with the Rb clock.

Keywords : BDS-3, Broadcast ephemeris, SISRE, Clock error,

\section{Introduction}

The BeiDou Satellite Navigation System (BDS) is a global satellite navigation system developed by China. Its development contains three stages: Demonstration Navigation 
Satellite System (BDS-1), Regional Navigation Satellite System (BDS-2), and Global BeiDou Navigation System (BDS-3). At present, the development trend of BDS continues to improve, and BeiDou-related services and industries are developing rapidly around the world. The full resolution of the compatibility of BDS with other global navigation satellite systems will make BDS more important in global positioning, navigation and timing (PNT) (China Satellite Navigation Office, 2019). Up to July, 2020, satellites working in-orbit in BeiDou navigation satellite system (BDS) consist of 15 BDS-2 satellites and 30 BDS-3 satellites, and most of the BDS-2 satellites clocks in-orbit are in the final phase, so it is essential to evaluate the performance of the satellites clocks and broadcast ephemeris. Thanks to the new inter-satellite link payloads on BDS-3 satellites, more than $98 \%$ of ephemerides and $93 \%$ of clock parameters are uploaded within one hour (Lv et al. 2019).

Many current research mainly focuses on the signal-in-space range error (SISRE), the satellite orbits and clock offsets calculated by broadcast ephemeris are compared with the precise orbit and clock offset products. With the contribution of BDS-3, the number of global average visible satellites has increased from 5.1 to 10.7 (Zhang et al. 2019), and all the results show that the accuracy level of BDS-3 is significantly higher than that of BDS-2 both in satellite orbit and in satellite clock offset. The corresponding RMS and STD of all BDS-3 satellite clock offsets are improved by $40.34 \%$ and $52.49 \%$ than that of BDS-2, respectively. Meanwhile, the mean RMS and STD are $1.78 \mathrm{~m}$ and $0.40 \mathrm{~m}$ for BDS-2 SISRE, $1.72 \mathrm{~m}$ and $0.34 \mathrm{~m}$ for BDS-2 orbit-only SISRE, $0.50 \mathrm{~m}$ and $0.14 \mathrm{~m}$ for BDS-3 SISRE, and $0.17 \mathrm{~m}$ and $0.04 \mathrm{~m}$ for BDS-3 orbit-only SISRE (Jiao et al. 2020). And similar results can also be found in other study. Yang found that the average RMS satellite clock error is 1.12 nanoseconds and the average SISRE is $0.44 \mathrm{~m}$ by evaluating 8 satellites of BDS-3 (Yang et al. 2019). Meanwhile, that of BDS-2 MEO satellites would be about $1 \mathrm{~m}$ (Wang et al. 2019). In terms of the clock offsets, BDS-3 satellites are equipped with high-precision domestic new rubidium clocks and passive 
hydrogen atomic clocks. Compared with the satellites of BDS-2, the performance of BDS-3 has great promotion (Mao et al. 2020).

This article mainly studies the accuracy of the BDS-3 broadcast ephemeris, and compares it with the BDS-2 broadcast ephemeris. We present an assessment of BDS-3 MEO satellites and BDS-2 three kinds of satellites broadcast orbit and clock offset accuracy from DOY 001-060, 2020. The precise products by the Wuhan University, which is one of the IGS MGEX analysis centers, are selected as the reference for the comparison. Finally, a standard single point positioning (SPP) test is used to evaluate the correctness of the results. Satellites positions and clock offsets derived from broadcast ephemeris are compared with precise orbit determination (POD) orbits and clock offsets. Then, the corresponding SISIRE is computed according to the SISRE definition.

In our paper, We first introduced the method of calculating satellite coordinates using broadcast ephemeris. It is worth noting that the caculation methods of MEO satellites, IGSO satellites and GEO satellites are slightly different. In addition, there are some considerations, such as the reference frame difference, antenna phase center correction, satellite clock error correction. After the satellite orbit error is obtained, the SISRE model is used to evaluate the broadcast ephemeris orbital accuracy and satellite clock error of BDS-2 and BDS-3 satellites, and the differences between them are compared.

\section{Methodology}

The evaluation of the broadcast of BDS-2 and BDS-3 satellites involves orbit errors and clock errors. Precise orbit and clock products obtained from WUM are used as true values to assess the performance of the broadcast ephemeris. The space segment of BDS is a hybrid constellation composed of MEO satellites, IGSO satellites and GEO satellites. However, due to the extremely small orbital inclination angle of GEO satellites, if the calculation is performed according to the orbital element method of 
MEO satellites and IGSO satellites, the normal equations are prone to singular matrices and calculation failures. Therefore, the calculation method for GEO satellites to calculate satellite positions from broadcast ephemeris is different from MEO satellites and IGSO satellites.

And the reference of the broadcast orbit and clock, as well as the precise clock, is the antenna phase center (APC), while the reference of the precise orbit is the center of mass $(\mathrm{CoM})$ of the satellite. Therefore, for the orbit comparison, the differences between CoM and APC need to be carefully corrected. For the clock comparison, the time group delay (TGD) caused by different signal or signal combinations also needs taking into account.

\section{Satellite position calculation method}

\section{BDS MEO and IGSO satellite coordinates (WGS84) calculation method}

The BDS satellite ephemeris provides 16 ephemeris parameters, including 1 reference moment, 6 Kepler orbit parameters at corresponding reference moments, and 9 orbital perturbation correction parameters. The ephemeris update period is $1 \mathrm{~h}$. The meaning of each parameter is as follows:

98 Table 1 16-parameter calculation model broadcast ephemeris parameters

\begin{tabular}{cc}
\hline parameter & Parameter meaning \\
\hline $\boldsymbol{t}_{\boldsymbol{o}}$ & Reference Time Ephemeris \\
$\sqrt{\boldsymbol{a}}$ & Square Root of the Semi-Major Axis \\
$\mathrm{e}$ & Eccentricity \\
$\boldsymbol{i}_{\mathbf{0}}$ & inclination Angel at Reference Time \\
$\mathbf{\Omega}_{\mathbf{0}}$ & Longitude of Ascending Node of Orbit \\
$\mathbf{\omega}$ & Plane at Weekly Epoch \\
$\mathbf{M}_{\mathbf{0}}$ & Argument of Perigee \\
$\Delta \mathbf{n}$ & Mean Anomaly at Reference Time \\
IDOT & Mean Motion Difference From \\
$\dot{\mathbf{\Omega}}$ & Computed Value \\
\hline
\end{tabular}




\begin{tabular}{|c|c|}
\hline \multirow{2}{*}{$C_{u s}$} & Amplitude of the Sine Harmonic \\
\hline & $\begin{array}{c}\text { Correction Term to the Argument of } \\
\text { Latitude }\end{array}$ \\
\hline & Amplitude of the Cosine Harmonic \\
\hline$C_{u c}$ & $\begin{array}{c}\text { Correction Term to the Argument of } \\
\text { Latitude }\end{array}$ \\
\hline$C_{r s}$ & $\begin{array}{l}\text { Amplitude of the Sine Harmonic } \\
\text { Correction Term to the Orbit Radius }\end{array}$ \\
\hline$C_{r c}$ & $\begin{array}{l}\text { Amplitude of the Cosine Harmonic } \\
\text { Correction Term to the Orbit Radius } \\
\text { Amplitude of the Sine Harmonic }\end{array}$ \\
\hline$C_{i s}$ & $\begin{array}{l}\text { Correction Term to the Angle of } \\
\text { Inclination }\end{array}$ \\
\hline$C_{i c}$ & $\begin{array}{l}\text { Amplitude of the Cosine Harmonic } \\
\text { Correction Term to the Angle of } \\
\text { Inclination }\end{array}$ \\
\hline
\end{tabular}

According to the ephemeris parameters to calculate the satellite position at any time

$100 \mathrm{t}$, the calculation steps are as follows:

101 Calculate the mean motion $\mathrm{n}_{0}$ of the satellite at the reference time $\mathrm{t}_{0}$

$$
n_{0}=\sqrt{\frac{\mu}{A^{3}}}
$$

In the formula: $\mu$ is the earth's gravitational constant in the BDCS coordinate

104 system, $\mu=3.986004418 \times 10^{14} \mathrm{~m}^{3} / \mathrm{s}^{2}, \sqrt{A}$ is the square root of the semi-major 105 axis given in the navigation message.

106 Using the difference $\Delta n$ between the satellite's average moving speed given in the 107 navigation message and the calculated value, calculated the corrected mean motion:

$$
\mathrm{n}=n_{0}+\Delta n
$$

109 Calculate the satellite mean anomaly $M_{k}$ at the moment of observation

$$
M_{k}=M_{0}+n\left(t-t_{o e}\right)
$$

111 Where, $t_{o e}$ is the ephemeris reference time given in the navigation message;

$112 M_{0}$ is the mean anomaly of the reference time $t_{o e}$ in the navigation message; t is BDT 113 at time of signal transmission; $t-t_{o e}$ is the total time difference, which must be 114 considered The start or end of the week transformation, that is: if $t-t_{o e}$ is greater 
115 than 302400 , subtract 604800 from $t-t_{o e}$; if $t-t_{o e}$ is less than -302400 , add 116604800 to $t-t_{o e}$.

117 Iterative calculation of the satellite near corner $\mathrm{E}$ at the moment of observation:

118 According to the eccentricity e given in the navigation message and the calculated 119 mean anomaly $M_{k}$, the Kepler's equation $E_{k}=M_{k}+e \sin E_{k}$ is used to calculate in 120 an iterative manner.

121 Solution method: First give the initial value of $\mathrm{E}: \mathrm{E}_{0}=\mathrm{M}$, and substitute the 122 above formula to solve the first iteration value. Stop the iteration when $\left|E_{k+1}-E_{k}\right|$ $<10^{-12}$

Calculate the true anomaly $f$ at the moment of observation:

$$
\left\{\begin{array}{l}
\sin v_{k}=\frac{\sqrt{1-e^{2}} \sin E_{k}}{1-e \cos E_{k}} \\
\cos v_{k}=\frac{\cos E_{k}-e}{1-e \cos E_{k}}
\end{array}\right.
$$

So the calculation formula for the true anomaly angle $v_{k}$ is:

$$
v_{k}=\tan ^{-1} \frac{\sqrt{1-e^{2}} \sin E_{k}}{\cos E_{k}-e}
$$

Calculate the argument of latitude $\Phi_{k}$ :

$$
\Phi_{k}=\omega+v_{k}
$$

131 message.

132 According to the perturbation parameters $C_{u c}, C_{u s}, C_{r c}, C_{r s}, C_{i c}, C_{i s}$ given in

133 the ephemeris, calculate the Argument of Latitude correction $\delta u_{k}$, the Radius 134 Correction $\delta r_{k}$, and the Inclination Correction $\delta i_{k}$.

$$
\left\{\begin{array}{l}
\delta u_{k}=C_{u c} \cos 2 \Phi_{k}+C_{u s} \sin 2 \Phi_{k} \\
\delta r_{k}=C_{r c} \cos 2 \Phi_{k}+C_{r s} \sin 2 \Phi_{k} \\
\delta i_{k}=C_{i c} \cos 2 \Phi_{k}+C_{i s} \sin 2 \Phi_{k}
\end{array}\right.
$$




$$
\left\{\begin{array}{c}
u_{k}=\Phi_{k}+\delta u_{k} \\
r_{k}=a\left(1-e \cos E_{k}\right)+\delta r_{k} \\
i_{k}=i_{0}+I D O T\left(t-t_{o e}\right)+\delta i_{k}
\end{array}\right.
$$

In the above formula: $\mathrm{a}$ is the long radius of the satellite orbit, $\mathrm{a}=(\sqrt{A})^{3}, \sqrt{A}$, $i_{0}$ and IDOT are respectively the square root of the semi-major axis and the orbital inclination at the reference moment given by the broadcast ephemeris parameters and the rate of change of orbital inclination.

Calculate the coordinates of the satellite in the Cartesian coordinate system of the orbital plane:

In the orbital plane Cartesian coordinate system (the coordinate origin is at the center of the earth), the $z_{0}$ axis is perpendicular to the orbital plane, the $x_{0}$ axis points to the ascending node, and $y_{0}$ is perpendicular to the $x_{0}$ axis in the orbit plane, forming a right-handed system. The satellite's planar Cartesian coordinates are:

$$
\left\{\begin{array}{l}
x_{0}=r \cos u_{k} \\
y_{0}=r \sin u_{k}
\end{array}\right.
$$

Calculate the longitude $\mathrm{L}$ of the ascending node at the time of observation:

$$
\mathrm{L}=\Omega_{0}+\left(\dot{\Omega}-\omega_{e}\right)\left(t-t_{o e}\right)-\omega_{e} t_{o e}
$$

In the above formula: $\dot{\Omega}$ and $\Omega_{0}$ are the rate of change of ascending node right ascension given by the broadcast ephemeris parameters and the ascending node right ascension calculated according to the reference moment; $\omega_{e}$ is the earth rotation rate in the CGCS2000 coordinate system $\omega_{e}=7.2921150 \times 10^{-5} \mathrm{rad} / \mathrm{s}$.

Calculate the coordinates of the satellite in the CGCS2000 coordinate system: First rotate the coordinate system as follows:

1) Rotate the angle $\omega_{s}$ clockwise around the $z_{0}$ axis to make the $x_{0}$ axis change from perigee to ascending node;

2) Rotate the $x_{0}$ axis clockwise by the angle $i_{k}$ to make the $z_{0}$ axis coincide with the sky axis; 
3 ) Rotate the angle $\Omega$ clockwise around the $z_{0}$ axis so that the $x_{0}$ axis coincides with

163 the $\mathrm{X}$ axis of the celestial coordinate system, thereby obtaining the coordinates of the 164 satellite in the celestial rectangular coordinate system.

165 Because when using BDS positioning, the position of the observation satellite and the 166 observation station should be in a unified coordinate system, and the coordinates in the 167 celestial coordinate system need to be converted to the earth space rectangular 168 coordinate system. The coordinate between the two points only on the $\mathrm{X}$ axis. When 169 the direction is different from the Greenwich star, so only one rotation is needed to find 170 the position of the satellite in the instantaneous earth coordinate system.

171 In summary, after knowing the longitude L of the ascending node and the 172 inclination $\mathrm{i}$ of the orbital plane, the position coordinates of the satellite in the ground173 fixed coordinate system can be easily obtained through two rotations.

174 The coordinates of the MEO/IGSO satellite in the CGCS2000 coordinate system are 175 $\left[\begin{array}{c}X \\ Y \\ Z\end{array}\right]=R_{Z}(-L) R_{X}\left(-i_{k}\right)\left[\begin{array}{c}x_{0} \\ y_{0} \\ 0\end{array}\right]=\left[\begin{array}{c}x_{0} \cos L-y_{0} \sin i_{k} \sin L \\ x_{0} \sin L+y_{0} \sin i_{k} \cos L \\ y_{0} \sin i_{k}\end{array}\right]$ In the formula, $\mathrm{L}$ is the ascension of the ascending node in the earth-solid system.

\section{BeiDou GEO satellite coordinates (WGS84) calculation method}

Due to the small inclination of the GEO orbit, the use of GPS ephemeris parameters

179 to fit the GEO satellite orbit may not converge due to the singularity of the matrix.

180 Literature proposes a coordinate rotation method to solve this problem. Specific steps 181 are as follows:

182 1) Transform the satellite ephemeris in the geo-fixed system to the quasi-J2000 183 coordinate system by rotating the GAST angle clockwise around the $\mathrm{Z}$ axis (the 184 Greenwich side time corresponding to the satellite ephemeris);

185 2) Rotate clockwise by $n^{\circ}$ (counterclockwise by $n^{\circ}$ ) around the X-axis or Y-axis in 186 the quasi-J2000 coordinate system to obtain the satellite ephemeris in the new inertial 187 system; 
188 Convert the new inertial system ephemeris obtained in the second step to the new 189 ground-fixed coordinate system by rotating the GAST angle counterclockwise around 190 the $\mathrm{Z}$ axis;

191 In the new ground-fixed coordinate system, the parameters of the broadcast ephemeris 192 are fitted according to the MEO calculation method.

193 In practical applications, when users calculate the longitude of the ascending node at 194 the instant of GEO satellite observation, the first step of rotation around the Z axis can 195 be omitted without considering the $\omega t_{k}$ term, that is, the satellite position can be 196 obtained by two-step coordinate transformation, reducing calculations the amount.

197 Ascension of the ascending node in the inertial frame is

$$
\mathrm{L}=\Omega_{0}+\dot{\Omega}\left(t-t_{o e}\right)-\omega_{e} t_{o e}
$$

1993 ) Solving the Greenwich side angle GAST of the instantaneous epoch will bring a lot 200 of computation to the receiver and bring inconvenience to the design of the receiver.

$$
\mathrm{GAST}=G A S T_{\text {toe }}+\omega t_{k}
$$

202 In the above formula, GAST toe represents the Greenwich side angle of the 203 reference $t_{o e}$ time, $\omega$ is the angular velocity of the earth's rotation, and $t_{k}=\mathrm{t}-t_{o e}$ 204 is the time difference from the instantaneous epoch to the reference epoch.

In order to avoid the above problems, rotate the reference plane under the inertial 206 system that coincides with the fixed coordinate system corresponding to the reference 207 time $t_{o e}$, which eliminates the need to calculate the more complicated $G A S T_{\text {toe }}$ term and only calculates the $\omega t_{k}$ part.

209 The GEO broadcast ephemeris parameters obtained by the coordinate rotation 210 method are fitted. When calculating the GEO satellite orbit, the user only needs to 211 calculate the satellite position according to the MEO satellite calculation method, and 212 then perform the corresponding coordinate inverse transformation process, and the 213 GEO satellite can be obtained. The position in the ground-fixed coordinate system, 214 namely the BeiDou satellite orbit algorithm 


$$
\left\{\begin{array}{c}
X_{G}=x_{0} \cos L-y_{0} \sin i \sin L \\
Y_{G}=x_{0} \sin L+y_{0} \sin i \cos L \\
Z_{G}=y_{0} \sin i
\end{array}\right.
$$

In the above formula, $\mathrm{L}$ is the right ascension of the ascending node in the inertial coordinate system

$$
\left[\begin{array}{l}
X \\
Y \\
Z
\end{array}\right]=R_{Z}\left(\omega_{e}\left(t-t_{o e}\right)\right) R_{X}\left(-5^{\circ}\right)\left[\begin{array}{c}
X_{G} \\
Y_{G} \\
Z_{G}
\end{array}\right]
$$

In the above formula,

$$
\begin{gathered}
R_{X}\left(-5^{\circ}\right)=\left[\begin{array}{ccc}
1 & 0 & 0 \\
0 & \cos \left(-5^{\circ}\right) & \sin \left(-5^{\circ}\right) \\
0 & -\sin \left(-5^{\circ}\right) & \cos \left(-5^{\circ}\right)
\end{array}\right] \\
R_{Z}\left(\omega_{e}\left(t-t_{o e}\right)\right)=\left[\begin{array}{ccc}
\cos \left(\omega_{e}\left(t-t_{o e}\right)\right) & \sin \left(\omega_{e}\left(t-t_{o e}\right)\right) & 0 \\
-\sin \left(\omega_{e}\left(t-t_{o e}\right)\right) & \cos \left(\omega_{e}\left(t-t_{o e}\right)\right) & 0 \\
0 & 0 & 1
\end{array}\right]
\end{gathered}
$$

\section{Precautions}

\section{Reference frame difference}

225 The Satellite positions derived from the BDS broadcast ephemeris are based on the BeiDou Coordinate System (BDCS), and the precise orbits are based on the

227 International Terrestrial Reference Frame (ITRF). Compared with the broadcast track

228 accuracy, the impact is negligible, and the impact of the difference between the two 229 frames can be ignored in the evaluation. However, it should be mentioned that Earth's 230 gravitational constant, GM, and Earth's rotation rate, $\omega$, and the flattening of the 231 ellipsoid adopted by the BeiDou differ from those used in GPS. For example, the value 232 of GM for BDS is $3.986004418 \times 10^{14}\left(\mathrm{~m}^{3} / \mathrm{s}^{2}\right)$ and the value for GPS is $2333.986005 \times 10^{14}\left(\mathrm{~m}^{3} / \mathrm{s}^{2}\right)$. The Earth rotation rate adopted for BDS is $7.2921150 \times$ $23410^{-5}$, and the value for GPS is $7.2921151467 \times 10^{-5}$ (Qin et al. 2019). If these 235 differences are not taken into account, it is possible to cause errors of tens of meters. 
237 The BDS satellite ephemeris provides 16 ephemeris parameters, including 1 reference

238 moment, 6 Kepler orbit parameters at corresponding reference moments, and 9 orbital

239 perturbation correction parameters. And the algorithm of the BDS MEO satellites and

240 GEO satellites broadcast ephemeris is described in detail in the interface documents

241 (China Satellite Navigation Office, 2017a, b; 2018; 2019a, b).

242 Antenna phase offset

243 The satellite position provided by the precision ephemeris is the center of mass (CoM)

244 of the satellite, while the position provided by the broadcast ephemeris is the antenna 245 phase center (APC). Staring from 7 January 2017, BDS changed the orbit reference 246 point from the CoM to the APC, which is the same as other GNSS systems. For WUM 247 products, the antenna phase center offset (PCO) used in broadcast ephemeris is 248 provided by the Test and Assessment Research Center of China Satellite Navigation 249 Office (TRAC-China Satellite Navigation Office), while PCO of BDS-2 are estimated 250 by Wuhan University.

\section{BDS Satellite Clock Offsets}

252 The clock difference between the broadcast ephemeris and precise ephemeris is a 253 systematic deviation. Considering that the indicator SISRE refers to the ranging error 254 from the user receiver to the phase center of the satellite antenna when evaluating the 255 accuracy of the broadcast ephemeris, there should be no systematic deviation. Therefore, 256 we must eliminate the systematic error of satellite clock error.

The hardware delay, for example the group delay, is part of the systemic error. The group delay (time group delay, TGD) parameter of the BDS broadcast ephemeris is one

259 of the important components for dual-frequency users to achieve positioning. The BDS 260 broadcast clock offset is based on the B3 frequency. The precision clock difference 261 between BDS-2 and BDS-3 is based on B1/B3 frequency. Therefore, in order to unify 262 the time base, processing needs to be performed when evaluating the satellite broadcast 263 ephemeris clock difference. 


$$
d_{t}=t_{B 3}-\frac{f_{1}^{2} T_{G D 1}}{f_{1}^{2}-f_{3}^{2}}
$$

In the above formula, $f_{1}$ and $f_{3}$ are the frequencies of B1 and B3 frequency points, respectively; $t_{B 3}$ is the satellite clock difference calculated from the broadcast ephemeris parameter; $T_{G D 1}$ is the group delay parameter of the BDS broadcast ephemeris (Jiao et al. 2020).

In this paper, a method is also used to calculate the mean value of the difference between the broadcast ephemeris and the precise ephemeris in one day for a single satellite. When comparing each ephemeris with the precise ephemeris, the difference between the clocks of different ephemeris and the mean value is used to eliminate the systematic deviation of the satellite clock.

\section{SISRE model}

SISRE is a common quantity used to assess the quality of broadcast ephemeris. The comm SISRE expression for multi-GNSS was defined by Montenbruck et al. (2018) and is represented as

$$
\text { SISRE }=\sqrt{\left(\omega_{1} R-T\right)^{2}+\omega_{2}^{2}\left(A^{2}+C^{2}\right)}
$$

Where $R, A$ and $C$ denote the orbit errors in radial, along-track and cross-track 281 direction, while $T$ represents clock errors. $\omega_{1}$ and $\omega_{2}$ are weight factors for the 282 global SISRE related to a specific constellation. If we neglect clock errors $T$, we obtain 283 the orbit-only SISRE formulation, that is,

$$
S I S R E_{\text {orbit-only }}=\sqrt{\omega_{1}^{2} R^{2}+\omega_{2}^{2}\left(A^{2}+C^{2}\right)}
$$

The detailed value of the weight factors for BDS-2 and BDS-3 satellites have been computed and presented by Montenbruck et al. (2015). 


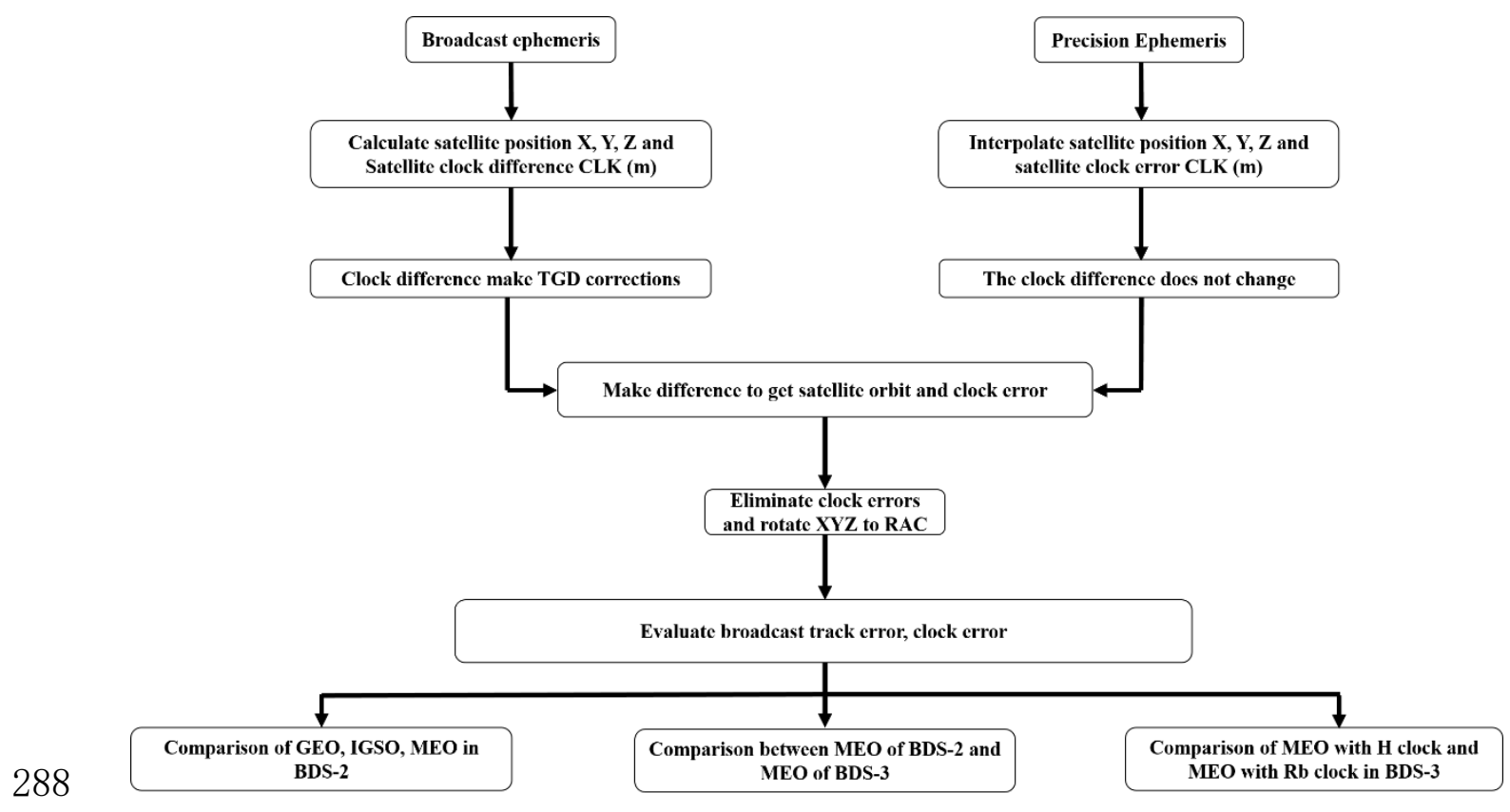

Fig. 1 Broadcast ephemeris accuracy assessment flowchart

$290 \quad$ Results and discussion

291 Table 2 BeiDou satellite navigation system satellite type

\begin{tabular}{|c|c|c|c|c|}
\hline $\begin{array}{c}\text { Satellite } \\
\text { system }\end{array}$ & \multicolumn{2}{|c|}{ Satellite type } & $\begin{array}{c}\text { Satellite } \\
\text { clock type }\end{array}$ & PRN \\
\hline \multirow{5}{*}{ BDS } & & GEO & \multirow{3}{*}{$\mathrm{Rb}$} & $\mathrm{C} 01 \sim \mathrm{C} 05$ \\
\hline & BDS-2 & IGSO & & $\mathrm{C} 6 \sim \mathrm{C} 10, \mathrm{C} 13, \mathrm{C} 16$ \\
\hline & & MEO & & C11、C12、C14 \\
\hline & \multirow[t]{2}{*}{ BDS-3 } & \multirow[t]{2}{*}{ MEO } & $\mathrm{Rb}$ & $\begin{array}{c}\mathrm{C} 19 \sim \mathrm{C} 24 、 \mathrm{C} 32 、 \mathrm{C} 33 、 \\
\mathrm{C} 36 、 \mathrm{C} 37\end{array}$ \\
\hline & & & $\mathrm{H}$ & $\mathrm{C} 25 \sim \mathrm{C} 30 、 \mathrm{C} 34, \mathrm{C} 35$ \\
\hline
\end{tabular}

\section{Comparison of GEO, IGSO, MEO in BDS-2}

294 Fig. 2, Fig. 3, Fig. 4 and Fig. 5 show the time series of the orbit error and clock error of

295 the three types of BDS satellite broadcasting in 60 days. Fig. 2, Fig. 3 and Fig. 4 are the 296 root mean square (RMS) orbit errors of a single GEO satellite (C04), a single IGSO 
satellite (C09) and a single MEO satellite (C11) in the R, A and C directions . Fig. 5

298 shows the root mean square of the three-dimensional orbit error, clock error and SISRE 299 time series of a single GEO satellite (C04), a single IGSO satellite (C09) and a single 300 MEO satellite (C11). It can be seen that the accuracy of BDS-2's MEO satellite 301 broadcast ephemeris is better than that of IGSO satellites and GEO satellites, and its 302 three-dimensional orbit error is basically around $2.6 \mathrm{~m}$.

303 For BDS-2 GEO satellites, it can be seen from Fig. 2 and Fig. 5 that the errors of GEO 304 satellites in the $\mathrm{R}$ and A directions are randomly distributed and stable. The fluctuation 305 range of GEO satellites in the R direction is within $1.8 \mathrm{~m}$, and the A direction is within $3062.3 \mathrm{~m}$. The orbit error of the GEO satellite in the $\mathrm{C}$ direction is within $7 \mathrm{~m}$. The three307 dimensional orbit error of GEO satellites is about $7.6 \mathrm{~m}$. The satellite clock error is about $3080.40 \mathrm{~m}$.

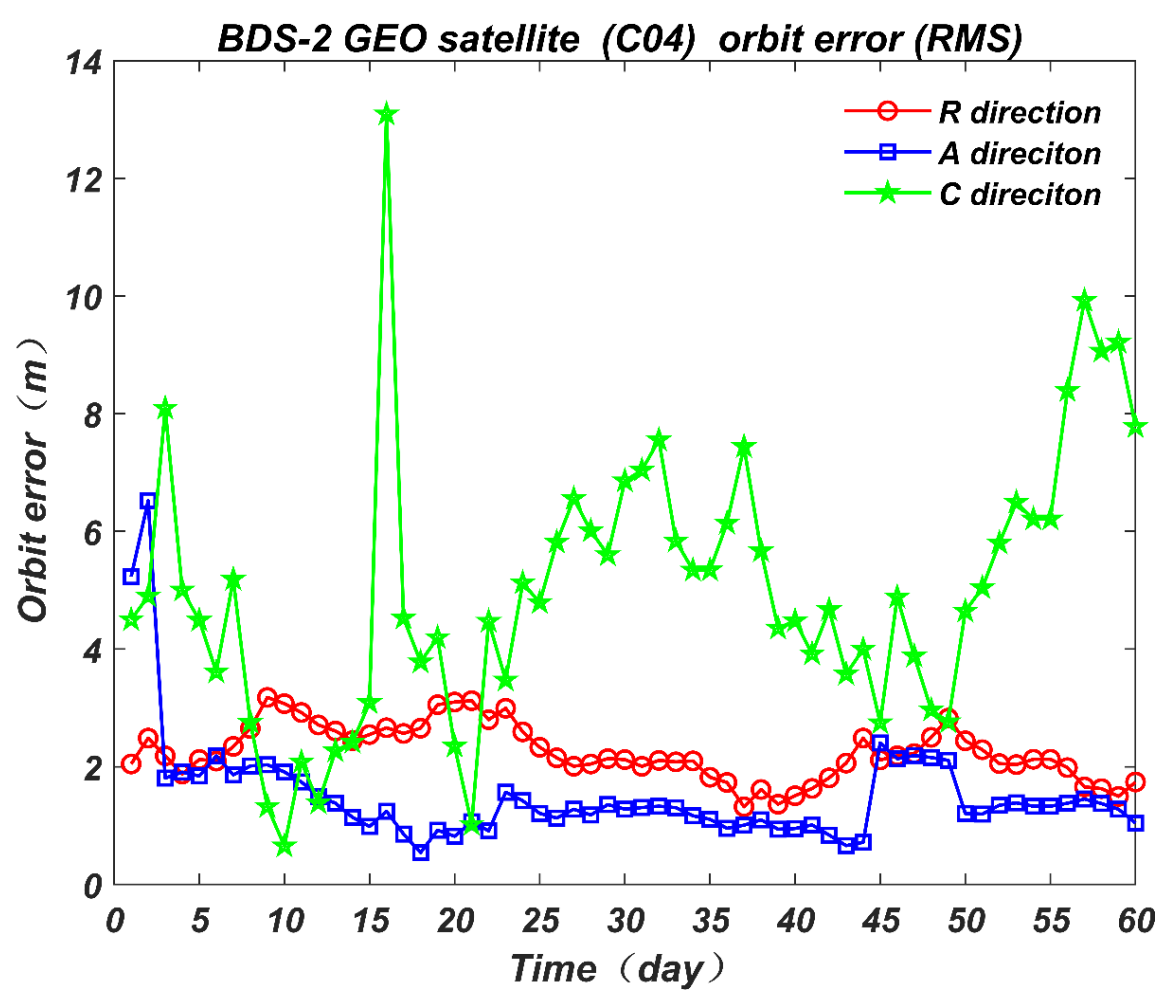

Fig. 2 BDS-2 GEO satellite (C04) orbit error (RMS) 


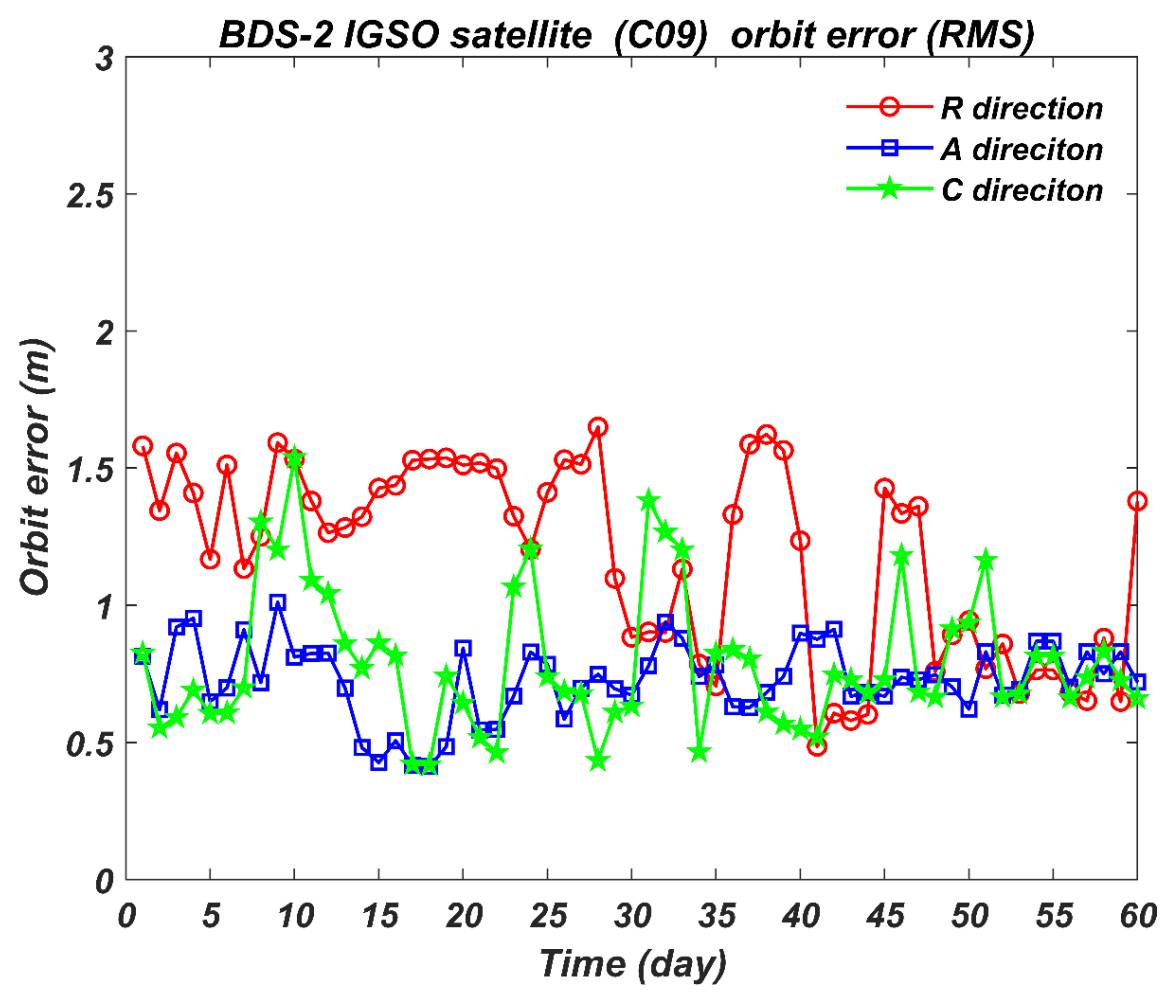

Fig. 3 BDS-2 IGSO satellite (C09) orbit error (RMS)

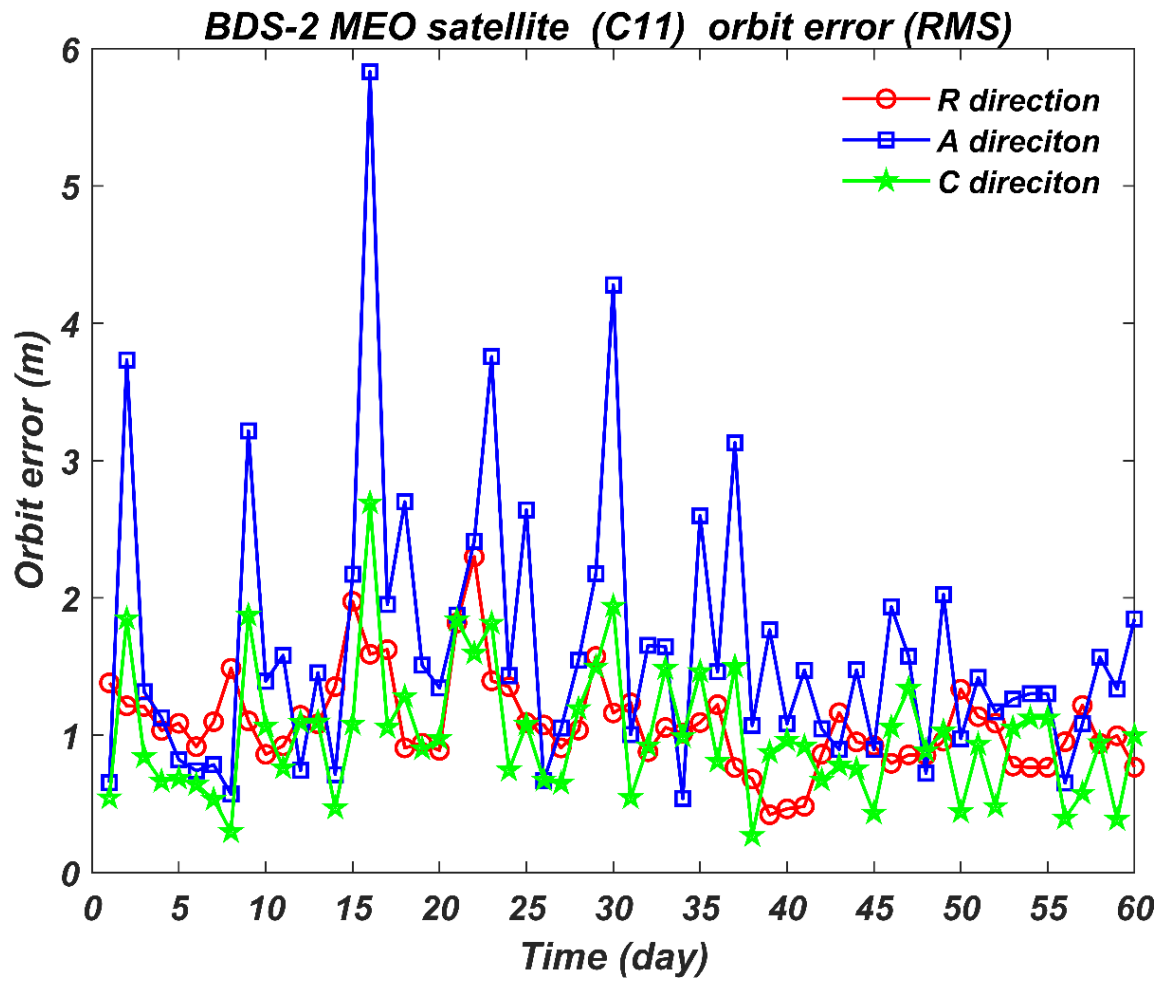

Fig. 4 BDS-2 MEO satellite (C11) orbit error (RMS) 
318 For the BDS-2 IGSO satellite, it can be seen from Fig. 3 and Fig.5 that the errors

319 in the $\mathrm{R}, \mathrm{A}$, and $\mathrm{C}$ directions of each satellite are randomly distributed, but the

320 fluctuations are stable. The fluctuation amplitude in the $\mathrm{R}$ and $\mathrm{C}$ directions is less than

$3211 \mathrm{~m}$, and the fluctuation amplitude in the A direction is not more than $0.5 \mathrm{~m}$. The three-

322 dimensional orbit error accuracy of IGSO satellite is about $2.4 \mathrm{~m}$. The satellite clock 323 error is about $0.59 \mathrm{~m}$.

324 For the BDS-2 MEO satellite, it can be seen from Fig. 4 and Fig. 5 that the R, A, 325 and $\mathrm{C}$ direction errors of each satellite are randomly distributed and not very stable, 326 with a lot of fluctuations, and the values are basically concentrated around 0 . The 327 accuracy of the three-dimensional orbit error of the MEO satellite is about $2.61 \mathrm{~m}$. The 328 satellite clock error is about $0.72 \mathrm{~m}$.
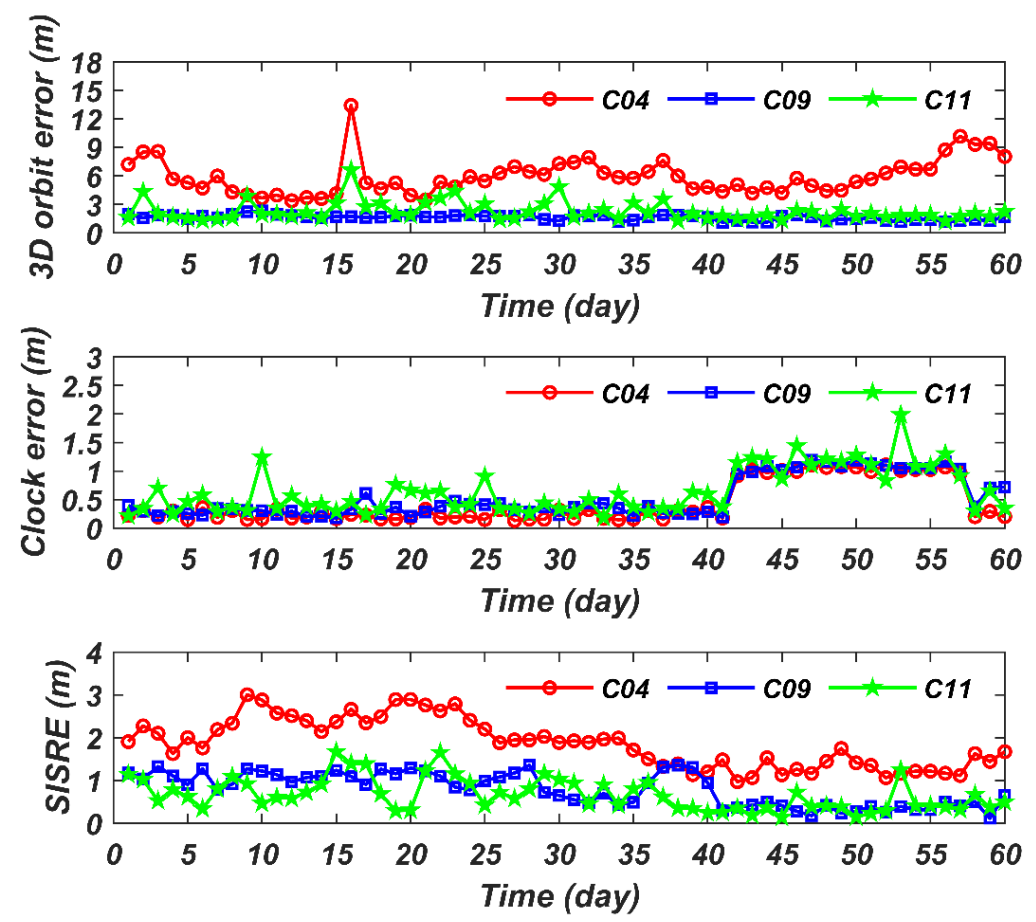

330 Fig. 5 BDS-2 satellite 3D orbit error (RMS), Clock error (RMS) and SISRE (RMS) 
It can be seen from Fig. 5 and Fig. 6 that the same Rb clock is used, and the root 334 mean square (RMS) of the orbit error of the MEO satellite of BDS-3 is smaller. The 335 three-dimensional orbit error of the MEO satellite of BDS-2 is about $2.7 \mathrm{~m}$, while the 336 three-dimensional orbit error of the MEO satellite of BDS-3 is about $0.5 \mathrm{~m}$.

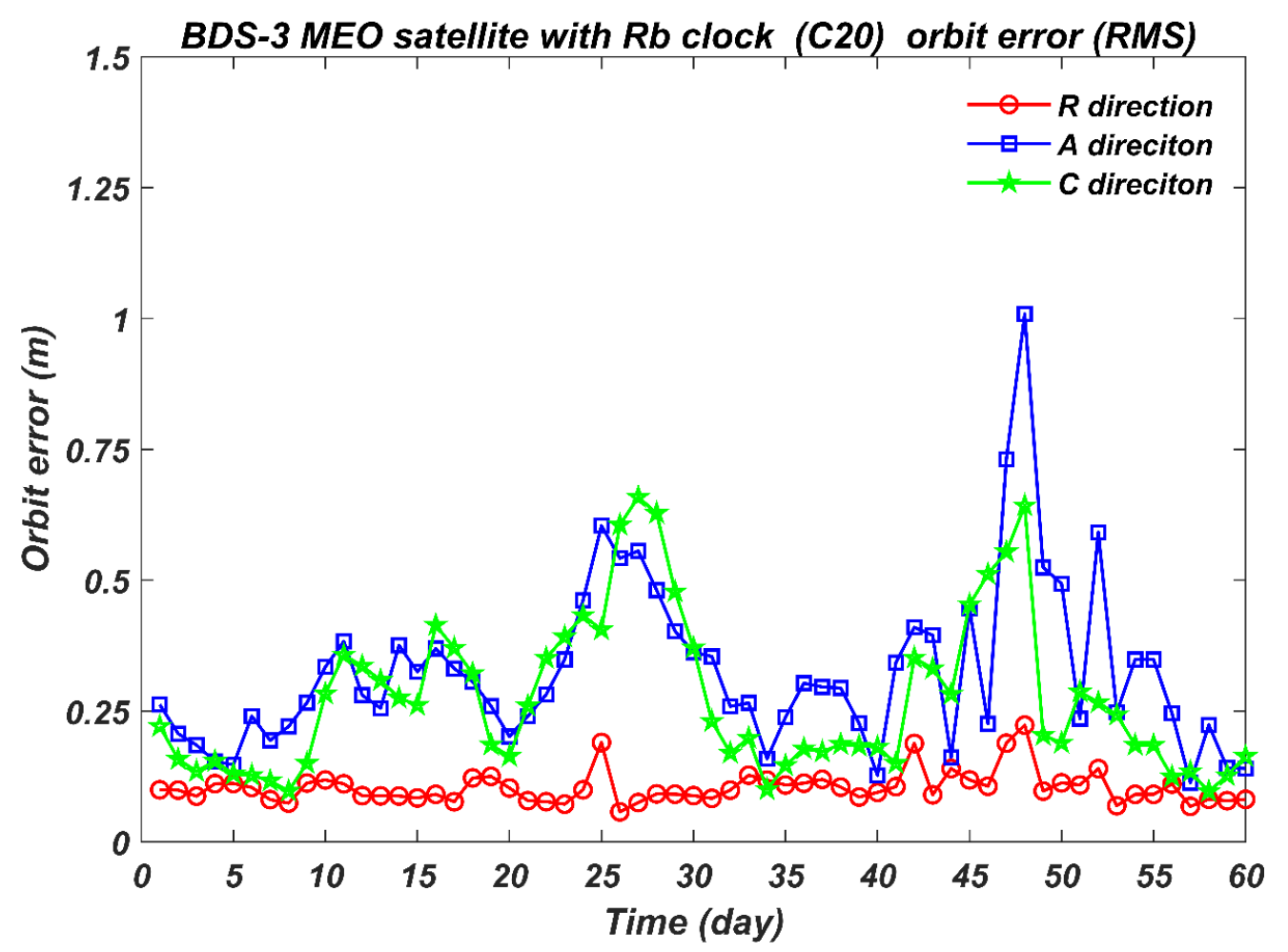

Fig. 6 BDS-3 MEO satellite with Rb clock (C20) orbit error (RMS) 


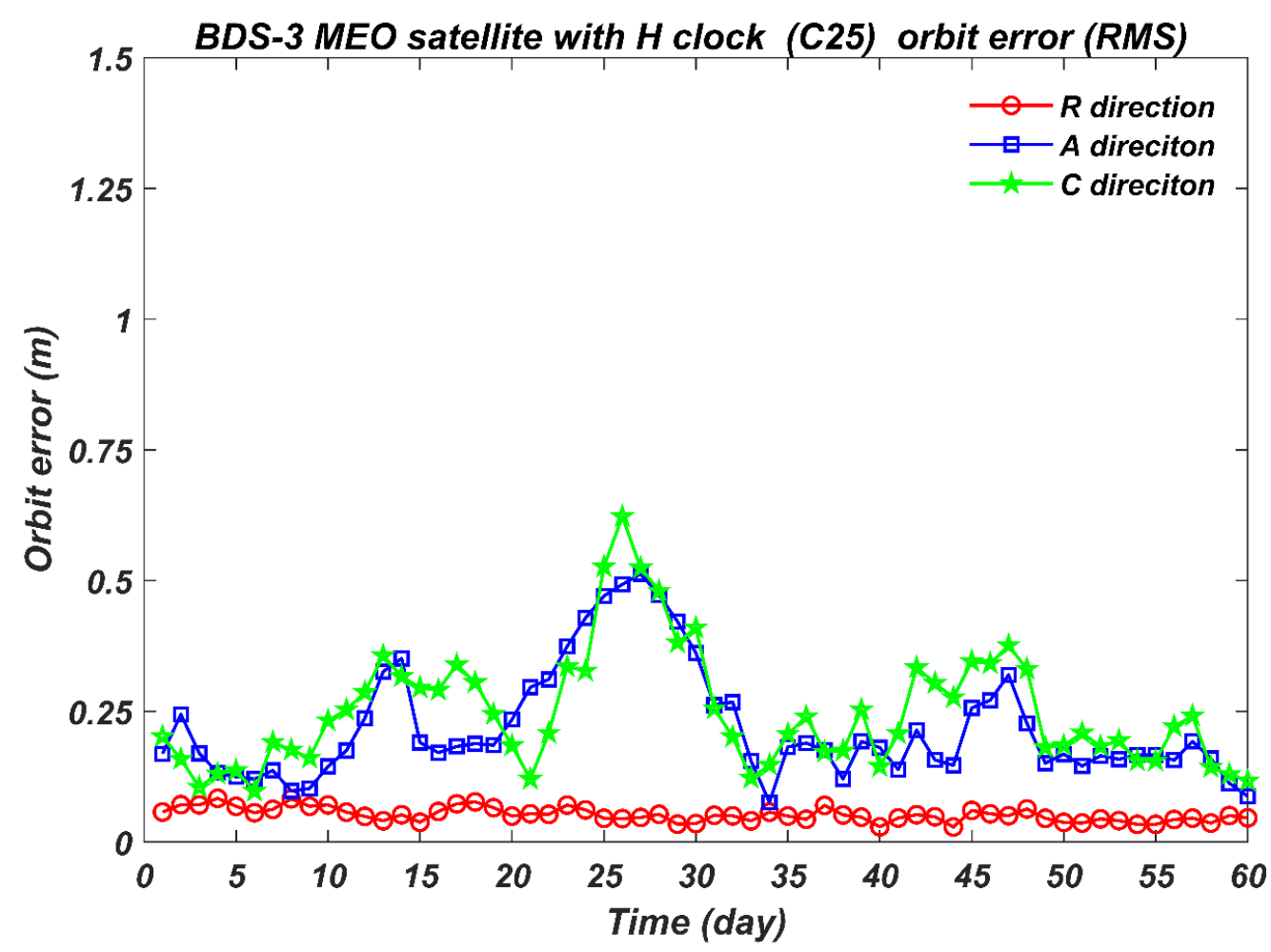

Fig. 7 BDS-3 MEO satellite with H clock (C25) orbit error (RMS) 

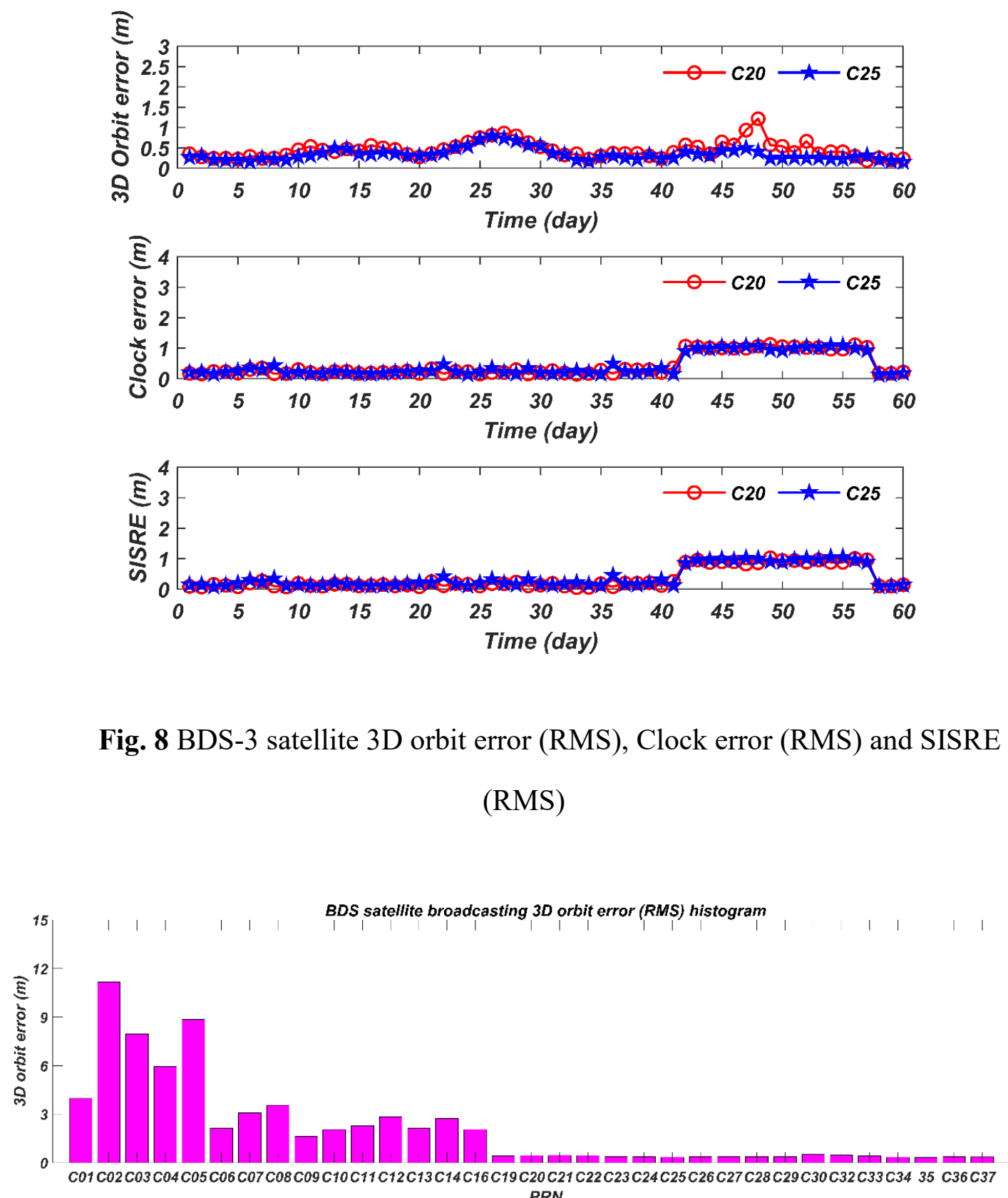

Fig. 9 BDS satellite broadcasting 3D orbit error (RMS) histogram

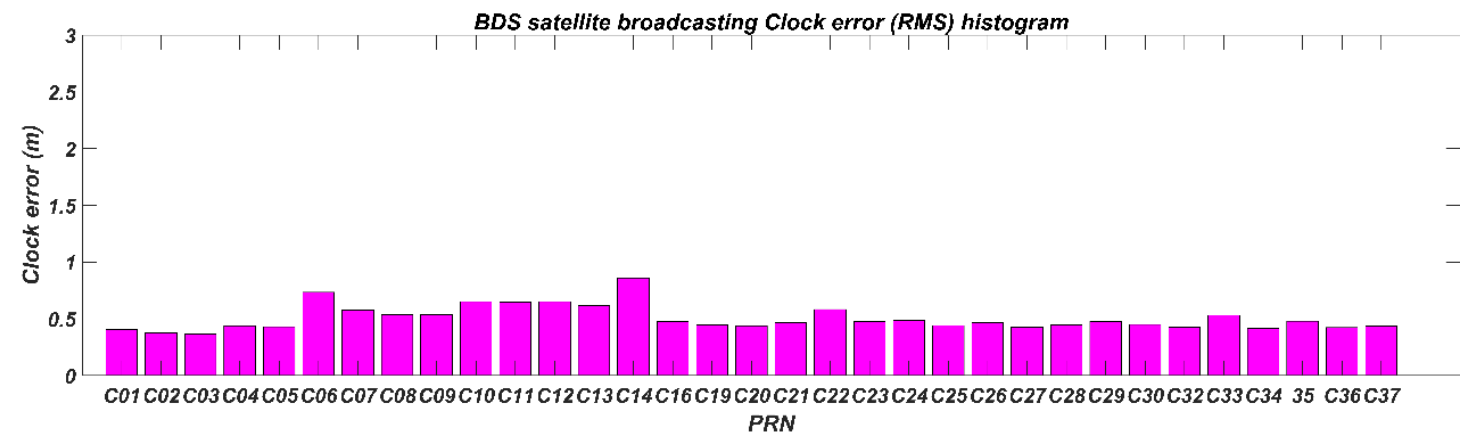

Fig. 10 BDS satellite broadcasting Clock error (RMS) histogram 


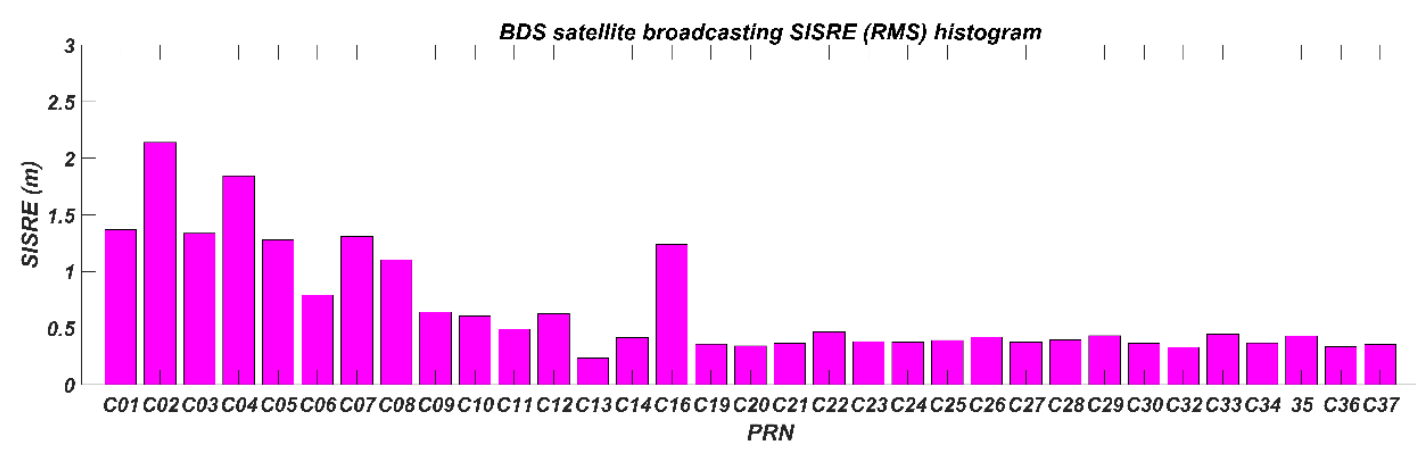

Fig. 11 BDS satellite broadcasting SISRE (RMS) histogram

364 From Fig. 9, Fig. 10 and Fig. 11, it can be seen that the root mean square error (RMS) 365 of the three-dimensional orbit of the BDS-3 satellite is better than that of the BDS-2 366 satellite, which is about $0.39 \mathrm{~m}$. The root mean square (RMS) difference of the clock 367 errors between BDS-3 and BDS-2 satellites is very small. The root mean square (RMS) 368 of SISRE of the BDS-3 satellite is generally better than that of the BDS-2 satellite, 369 which is about $0.39 \mathrm{~m}$.

371 Table 3 Average results of various satellites (RMS)

\begin{tabular}{|c|c|c|c|c|c|c|}
\hline \multirow[b]{2}{*}{ Satellite type } & \multicolumn{3}{|c|}{$\begin{array}{l}\text { Direction error } \\
(\mathbf{m})\end{array}$} & \multirow{2}{*}{$\begin{array}{c}\text { Three- } \\
\text { dimensional } \\
\text { (m) }\end{array}$} & \multirow{2}{*}{$\begin{array}{c}\text { Clock } \\
\text { difference } \\
(\mathbf{m})\end{array}$} & \multirow[b]{2}{*}{$\begin{array}{l}\text { SISRE } \\
(\mathbf{m})\end{array}$} \\
\hline & $\begin{array}{l}\text { Dire } \\
\text { ction } \\
\mathbf{R}\end{array}$ & $\begin{array}{c}\text { Direc } \\
\text { tion } \\
\text { A }\end{array}$ & $\begin{array}{c}\text { Direc } \\
\text { tion } \\
\text { C }\end{array}$ & & & \\
\hline $\begin{array}{c}\text { BDS-2 } \\
\text { GEO }\end{array}$ & 1.86 & 2.21 & 6.78 & 7.57 & 0.40 & 1.59 \\
\hline $\begin{array}{c}\text { BDS-2 } \\
\text { IGSO }\end{array}$ & 1.43 & 1.03 & 1.43 & 2.37 & 0.59 & 0.85 \\
\hline $\begin{array}{l}\text { BDS-2 } \\
\text { MEO }\end{array}$ & 1.14 & 1.98 & 1.10 & 2.61 & 0.72 & 0.51 \\
\hline BDS-2 ALL & 1.48 & 1.74 & 3.11 & 4.18 & 0.57 & 0.98 \\
\hline BDS-3 Rb & 0.10 & 0.28 & 0.27 & 0.41 & 0.47 & 0.38 \\
\hline MEO $\quad \mathrm{H}$ & 0.06 & 0.26 & 0.25 & 0.37 & 0.45 & 0.40 \\
\hline BDS-3 ALL & 0.08 & 0.27 & 0.26 & 0.39 & 0.46 & 0.39 \\
\hline
\end{tabular}




\section{Conclusion}

This article uses precision ephemeris to evaluate the accuracy of BDS-2 and BDS3 satellite broadcast ephemeris, sets up three sets of experiments, and uses standard single-point positioning results to check the veracity of the conclusions. The three sets of experiments respectively show:

1) The three-dimensional orbit errors of MEO satellites and IGSO satellites in BDS-2 are both better than those of GEO satellites. The three-dimensional orbit error of GEO satellites is about $7.6 \mathrm{~m}$, the error in the R direction is better than $2 \mathrm{~m}$, and the

381 error in the $\mathrm{C}$ direction is the largest, about $7.0 \mathrm{~m}$. The errors of MEO satellites and 382 IGSO satellites in all directions are basically the same, and the three-dimensional orbit error is better than $2.7 \mathrm{~m}$.

2) The orbit error of the BDS-3 satellite in each direction is better than $0.5 \mathrm{~m}$, and the three-dimensional orbit error is about $0.39 \mathrm{~m}$, which is obviously better than that of the BDS-2 satellite. The substantial increase in the orbital accuracy of the BDS-3 satellite may be related to the increase in interstellar links.

3) The clock difference of BDS-3 satellites equipped with $\mathrm{H}$ clock is better than that of satellites equipped with Rb clock, and the clock difference is about $0.45 \mathrm{~m}$. This is related to the type and nature of the satellite clock. The stability of the $\mathrm{H}$ clock is

391 better than that of the $\mathrm{Rb}$ type, and the clock difference produced is smaller.

\section{Declarations}

\section{List of abbreviations}

None.

\section{Ethics approval and consent to participate}

$396 \quad$ Not applicable.

\section{Consent for publication}




\section{Competing interests}

$$
\text { None }
$$

\section{Funding}

$404 \quad$ No funds are received.

\section{Authors' contributions} and analysis of thesis, debugging of experimental programs, and guidance of ideas.

\section{Acknowledgements}

None.

\section{Authors' information}

Haojun Jian is currently a third-year undergraduate in the School of Geodesy and

412 Geomatics of Wuhan University. His main research interests include GNSS navigation 413 data processing and algorithms.

414 Yishi Wang is currently a graduate student at Wuhan University. He obtained his 415 B.Sc. degree in the School of Geodesy and Geomatics of Wuhan University in 2015. 416 His main research interests include precise point positioning (PPP),-Satellite orbit 417 determination.

418 Shoujian Zhang is currently an associate professor at Wuhan University. He 419 obtained his B.Sc., Master, and Ph.D. degrees with distinction in Geodesy and 420 Engineering Surveying at the School of Geodesy and Geomatics in Wuhan University 421 in 2004, 2006 and 2010. His main research interests include precise point positioning (PPP), GNSS/INS navigations. 


\section{References}

China Satellite Navigation Office. (2017a) BeiDou navigation satellite system signal in space interface control document open service signal B2a (Version 1.0). https ://www.BeiDou.gov.cn/xt/gfxz/201712/P0201712267423573641 74.pdf. (Accessed 24 Oct 2019)

China Satellite Navigation Office. (2017b) BeiDou navigation satellite system signal in space interface control document open service signal B1C (Version 1.0). https://www.BeiDou.gov.cn/xt/gfxz/201712/P020171226 741342013031.pdf. (Accessed 24 Oct 2019)

China Satellite Navigation Office. (2018) BeiDou navigation satellite system signal in space interface control document open service signal B3I (Version 1.0). https ://www.BeiDou.gov.cn/xt/gfxz/201802/P0201802096236014011 89.pdf. (Accessed 24 Oct 2019)

China Satellite Navigation Office. BeiDou navigation satellite system signal in space interface control document open service signal B1I (Version 3.0). https ://www.BeiDou.gov.cn/xt/gfxz/201902/P0201902275936211424 75.pdf. (Accessed 24 Oct 2019)

China Satellite Navigation Office. (2019b) BeiDou navigation satellite system signal in space interface control document open service signal B2b (Beta Version). https://www.BeiDou.gov.cn/xt/gfxz/201912/P020191227325641753862.pdf. (Accessed 24 Oct 2019)

China Satellite Navigation Office. (2019) Development of the BeiDou Navigation Satellite System (Version 3.0). https://www.BeiDou.gov.cn/xt/gfxz/201812/P020190117356387956569.pdf. (Accessed 24 Dec 2020)

Choi Jin Haeng, Kim Gimin, Lim Deok Won, Park Chandeok (2020) Study on Optimal Broadcast Ephermeris Parameters for GEO/IGSO Navigation Satellites. Sensors. doi:10.3390/s20226544 
Dai P, Ge Y, Qin W, Yang X (2019) BDS-3 Time Group Delay and Its Effect on Standard Point Positioning. Remote Sensing. doi:10.3390/rs11151819.

Jiao G, Song S, Liu Y, Su K, Cheng N, Wang S (2020) Analysis and Assessment of BDS-2 and BDS-3 Broadcast Ephemeris: Accuracy, the Datum of Broadcast Clocks and Its Impact on Single Point Positioning. Remote Sensing. doi:10.3390/rs12132081.

Lv Y, Geng T, Zhao Q, Xie X, Zhou R (2019) Initial assessment of BDS-3 preliminary system signal-in-space range error. GPS Solution 24(10):423-434. doi:10.1007/s10291-019-0928-x.

Montenbruck O, Steigenberger P, Hauschild A (2015) Broadcast versus precise ephemerides: a multi-GNSS perspective. GPS Solut (2015) 19:321-333. doi:10.1007/s10291-014-0390-8.

Mao Y, Wang Q, Hu C, Yang H, Zhang M (2020) Analysis of the Characterization for BDS-3 Satellite Clock Error. Geomatics and Information Science of Wuhan University 2020,45(01):53-61. doi:10.13203/j.whugis20180224, (in Chinese with English abstract)

Wang H, Jia X, Li D, et al (2019) Accuracy assessment and analysis of broadcast ephemeris of BDS-3 satellites. Journal of Navigation and Positioning,2019,7(4): 60-63. doi:10.16547/j.cnki.10-1096.20190411, (in Chinese with English abstract)

Qin X, Yang Y; Cui X (2019) Influence of ellipsoid constants on interoperability of broadcast ephemeris computation between BeiDou and GPS. Geomatics and Information Science of Wuhan University 2015,40(9):1237-1241. doi:10.13203/j.whugis20130475, (in Chinese with English abstract)

Xie X, Geng T, Zhao Q, Lv Y, Cai H, Liu J (2020) Orbit and clock analysis of BDS-3 satellites using inter-satellite link observations. Journal of Geodesy 94(7):1-18. doi:10.1007/s00190-020-01394-4.

Yang Y, Gao W, Guo S, Mao Y, Yang Y (2019) Introduction to BeiDou-3 navigation 
satellite system. Navgation 2019,66(1):7-18. doi:10.1002/navi.291.

481 Zhang Y, Kubo N., Chen J, Chu F, Wang A, Wang J (2019) Apparent clock and TGD biases between BDS-2 and BDS-3. GPS Solution 2019, 24(10):433-441. doi:10.1007/s10291-019-0933-0.

484 Zhang Y, Kubo N., Chen J, Wang J, Wang H (2019) Initial Positioning Assessment of 485 BDS New Satellites and New Signals. Remote Sensing. doi:10.3390/rs11111320. 
Figures

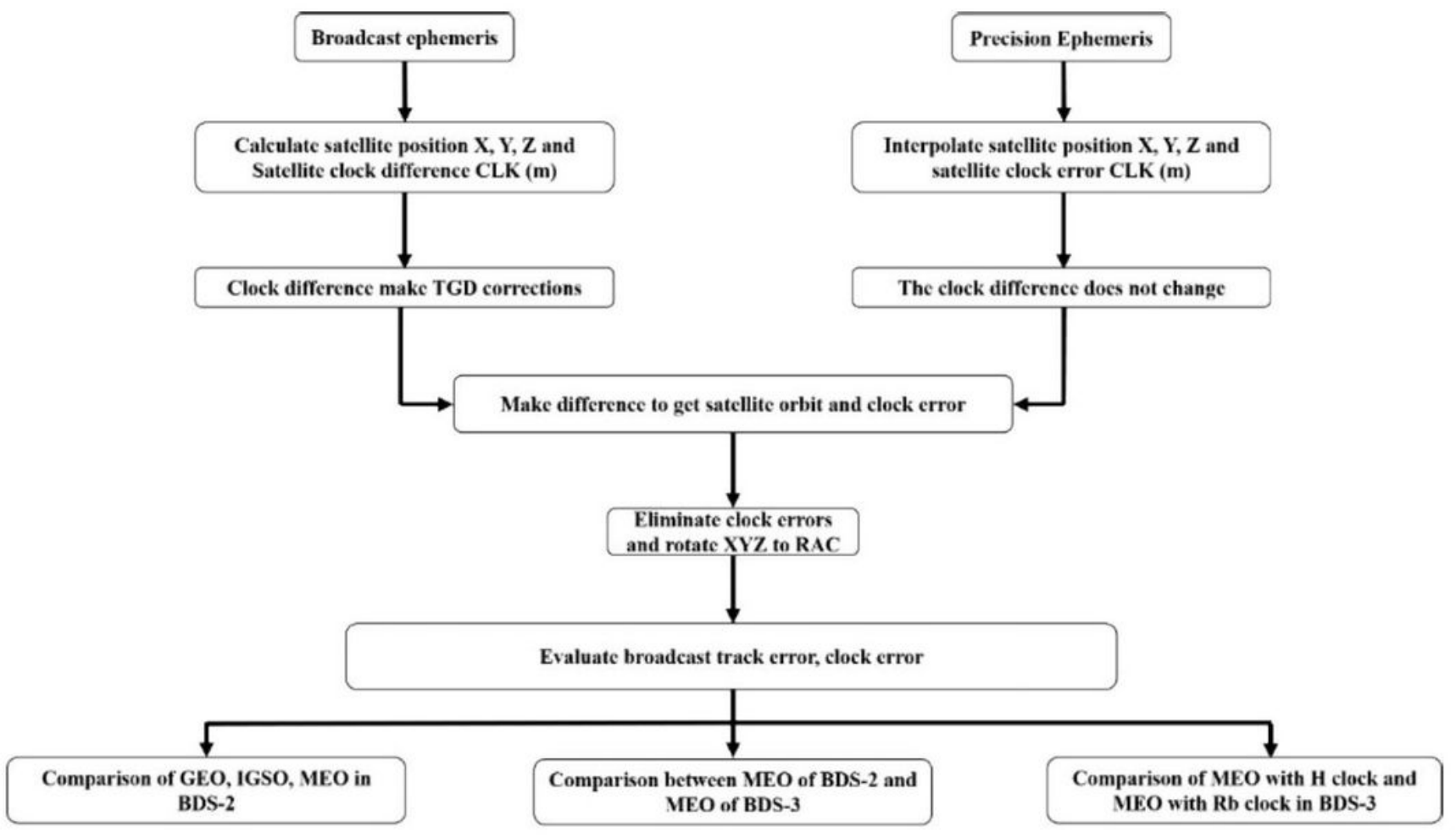

Figure 1

Broadcast ephemeris accuracy assessment flowchart 


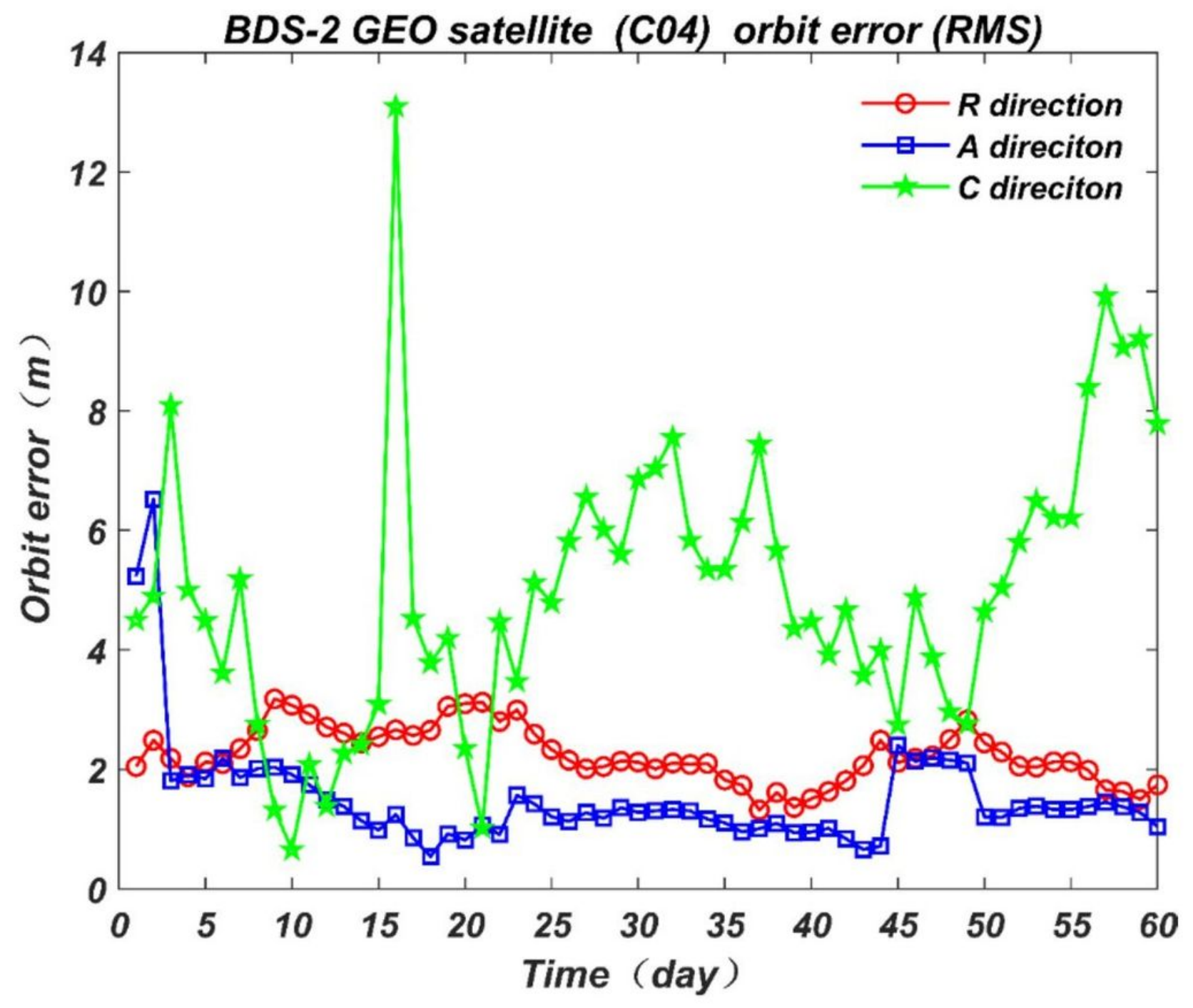

Figure 2

BDS-2 GEO satellite (C04) orbit error (RMS) 


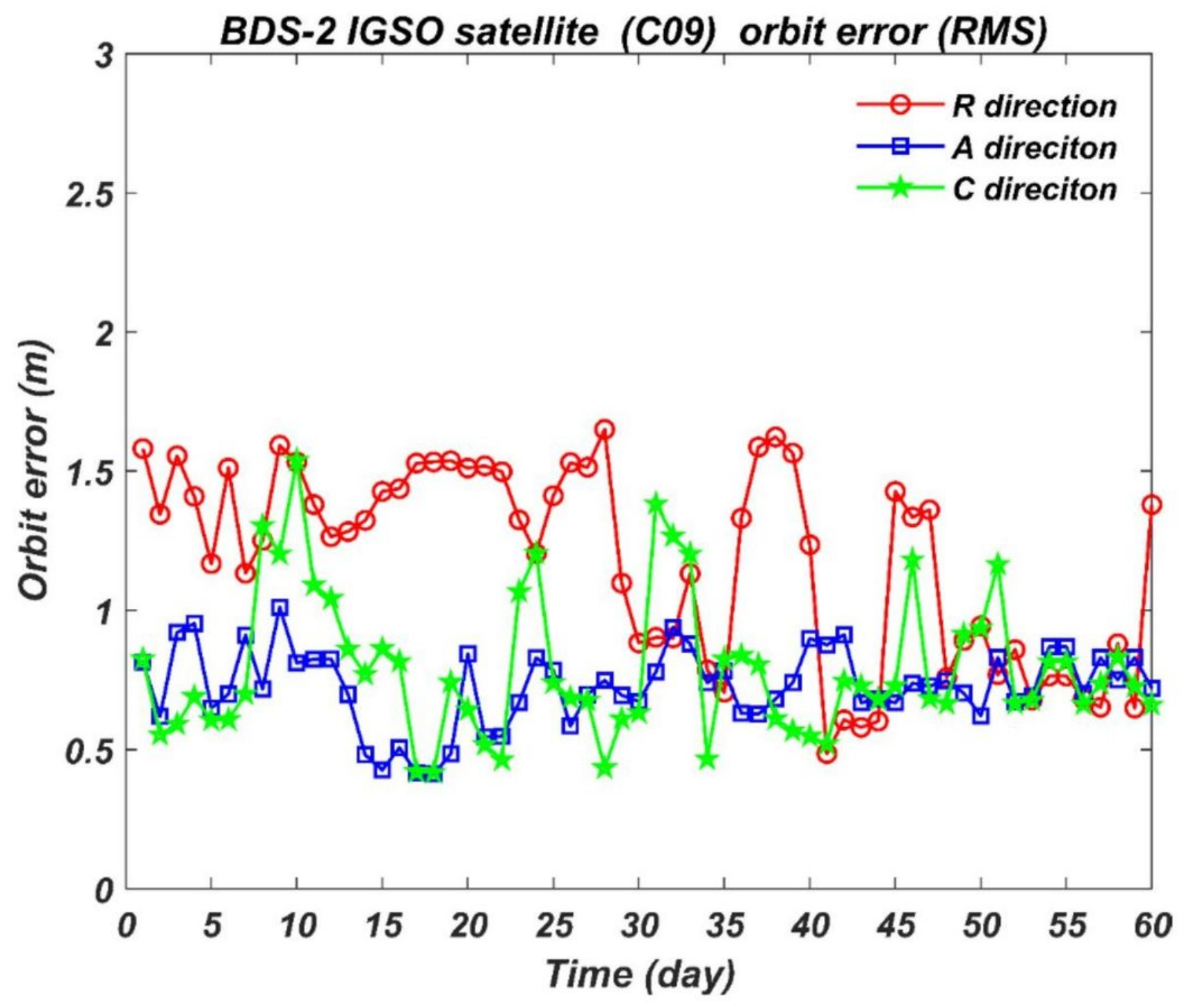

Figure 3

BDS-2 IGSO satellite (C09) orbit error (RMS) 


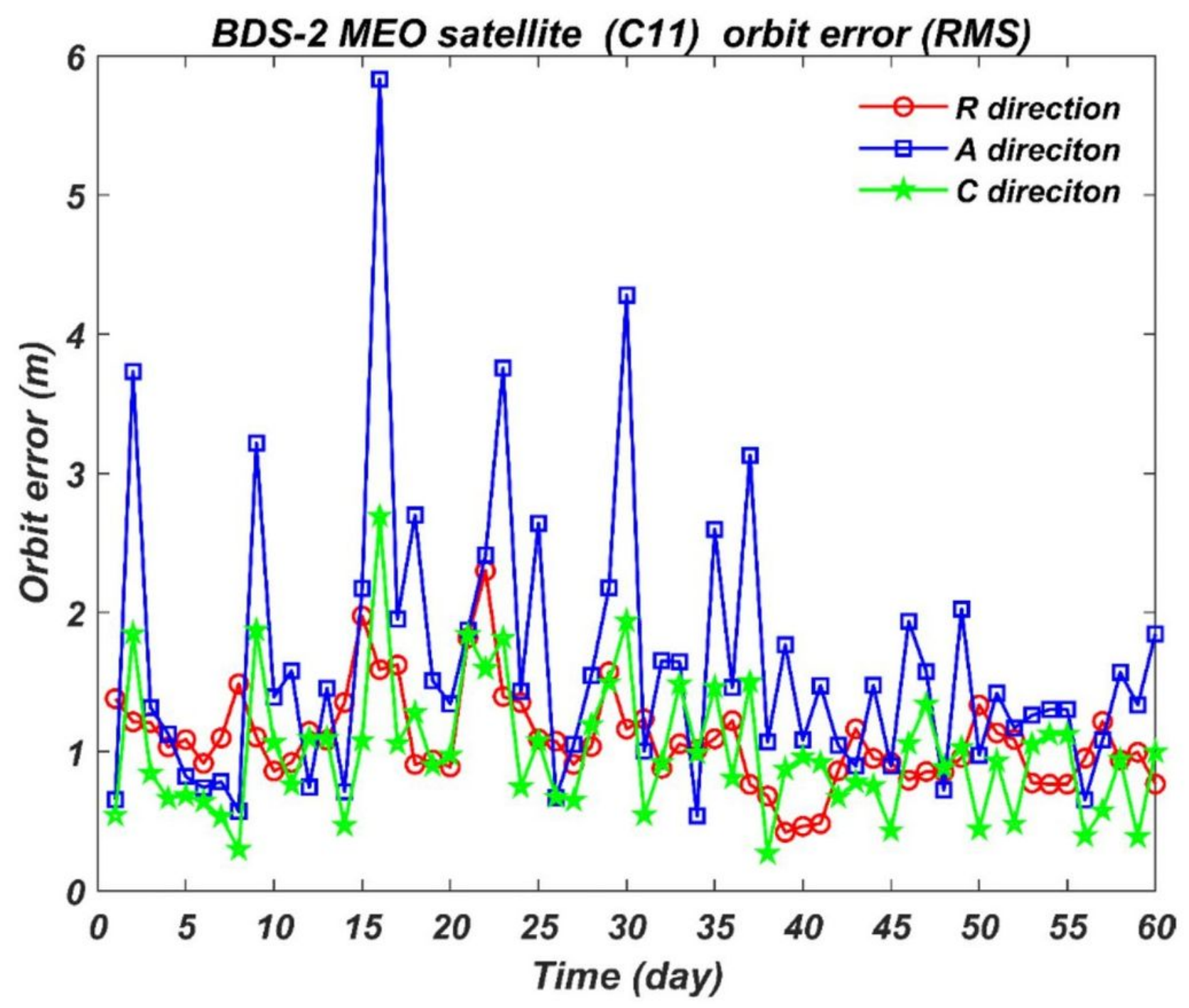

Figure 4

BDS-2 MEO satellite (C11) orbit error (RMS) 

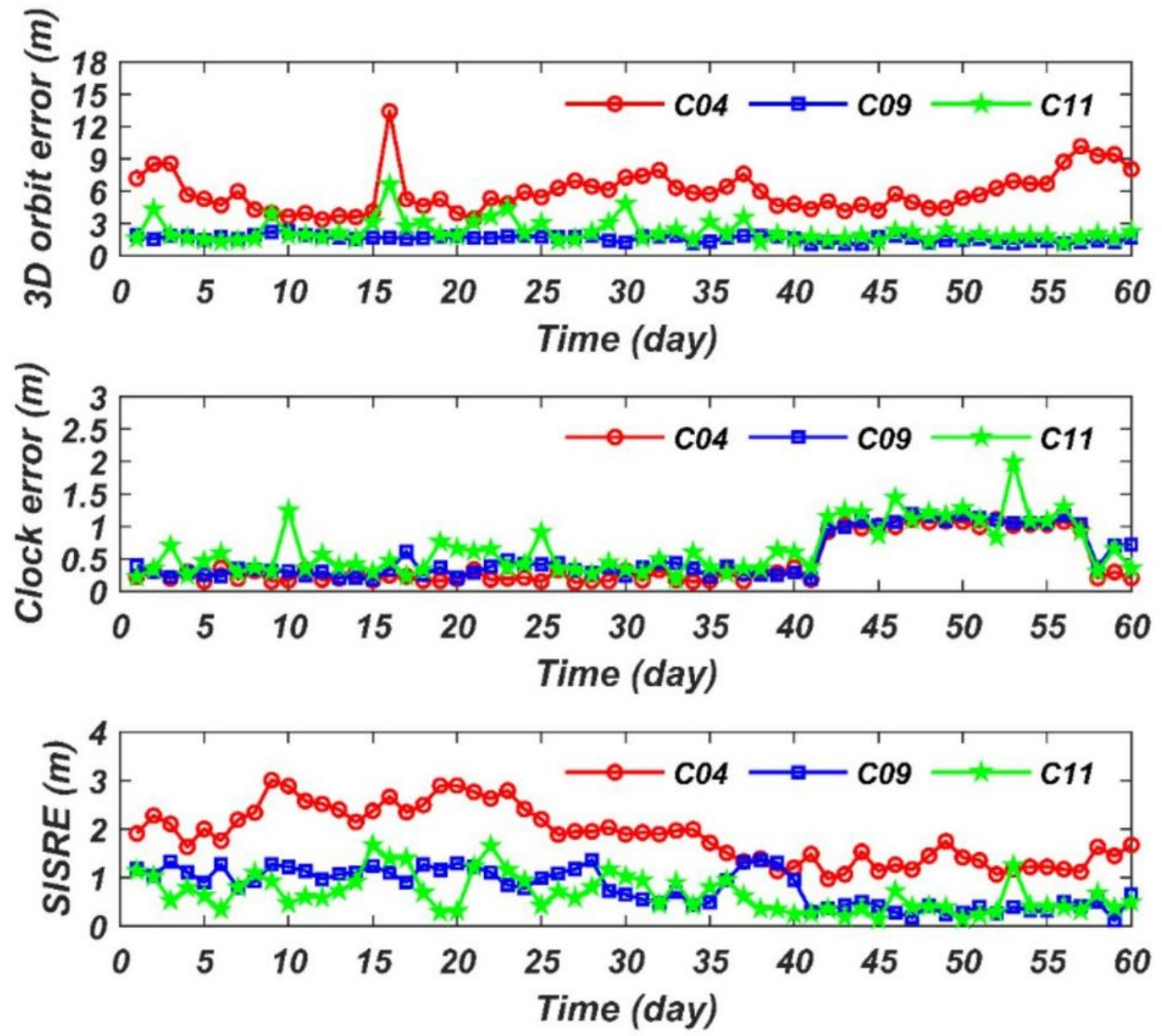

Figure 5

BDS-2 satellite 3D orbit error (RMS), Clock error (RMS) and SISRE (RMS) 


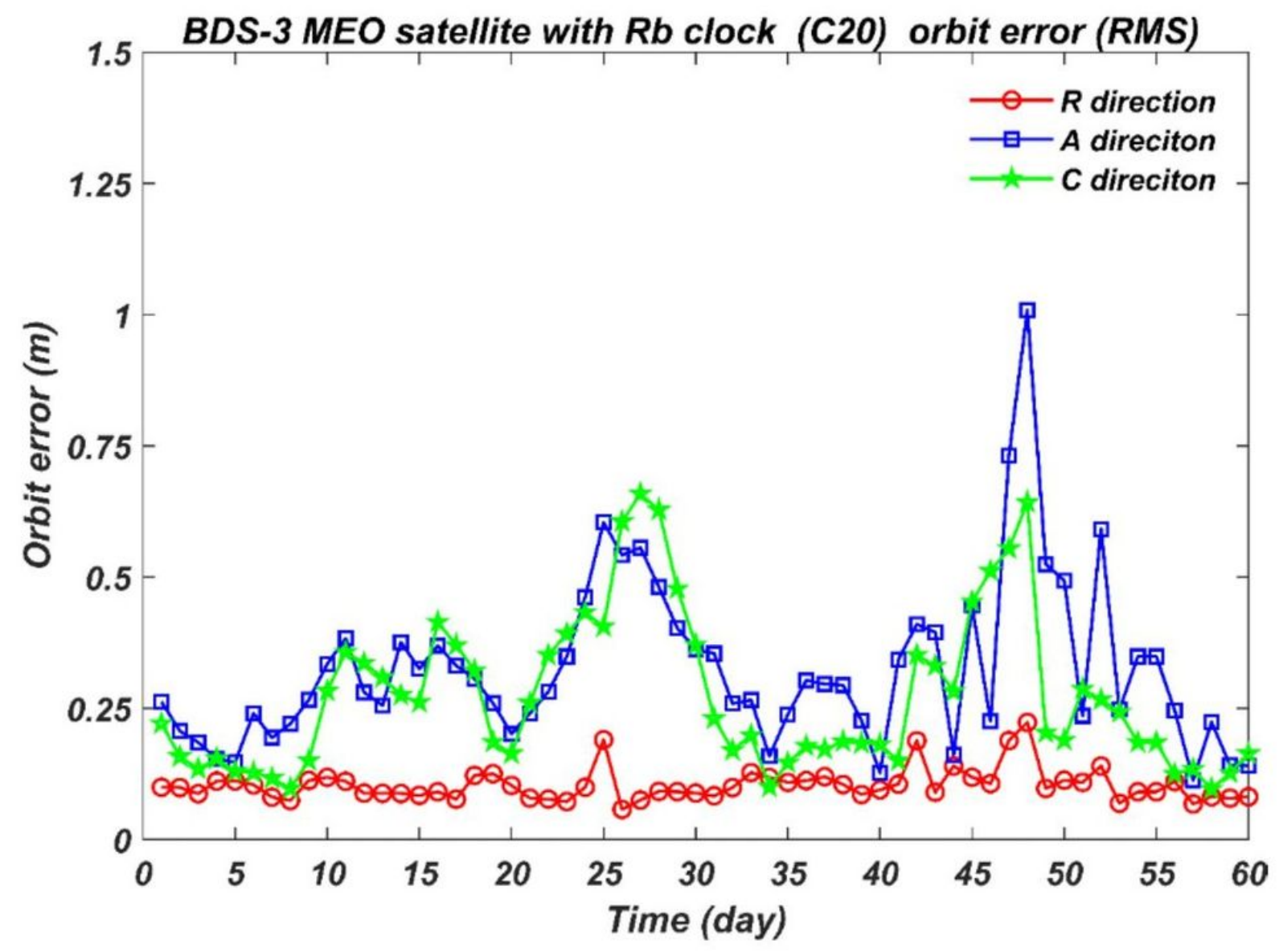

Figure 6

BDS-3 MEO satellite with Rb clock (C20) orbit error (RMS) 


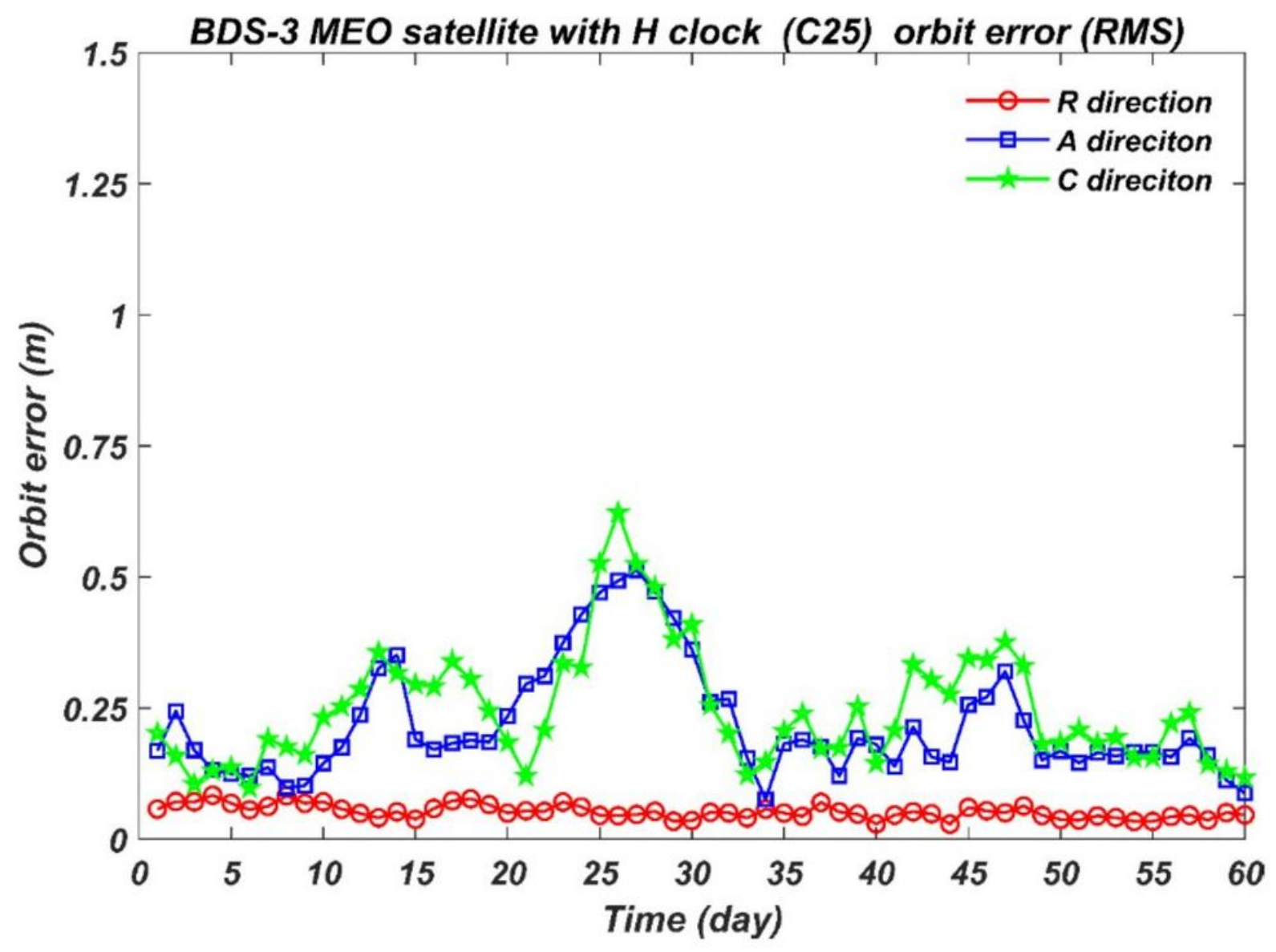

Figure 7

BDS-3 MEO satellite with H clock (C25) orbit error (RMS) 

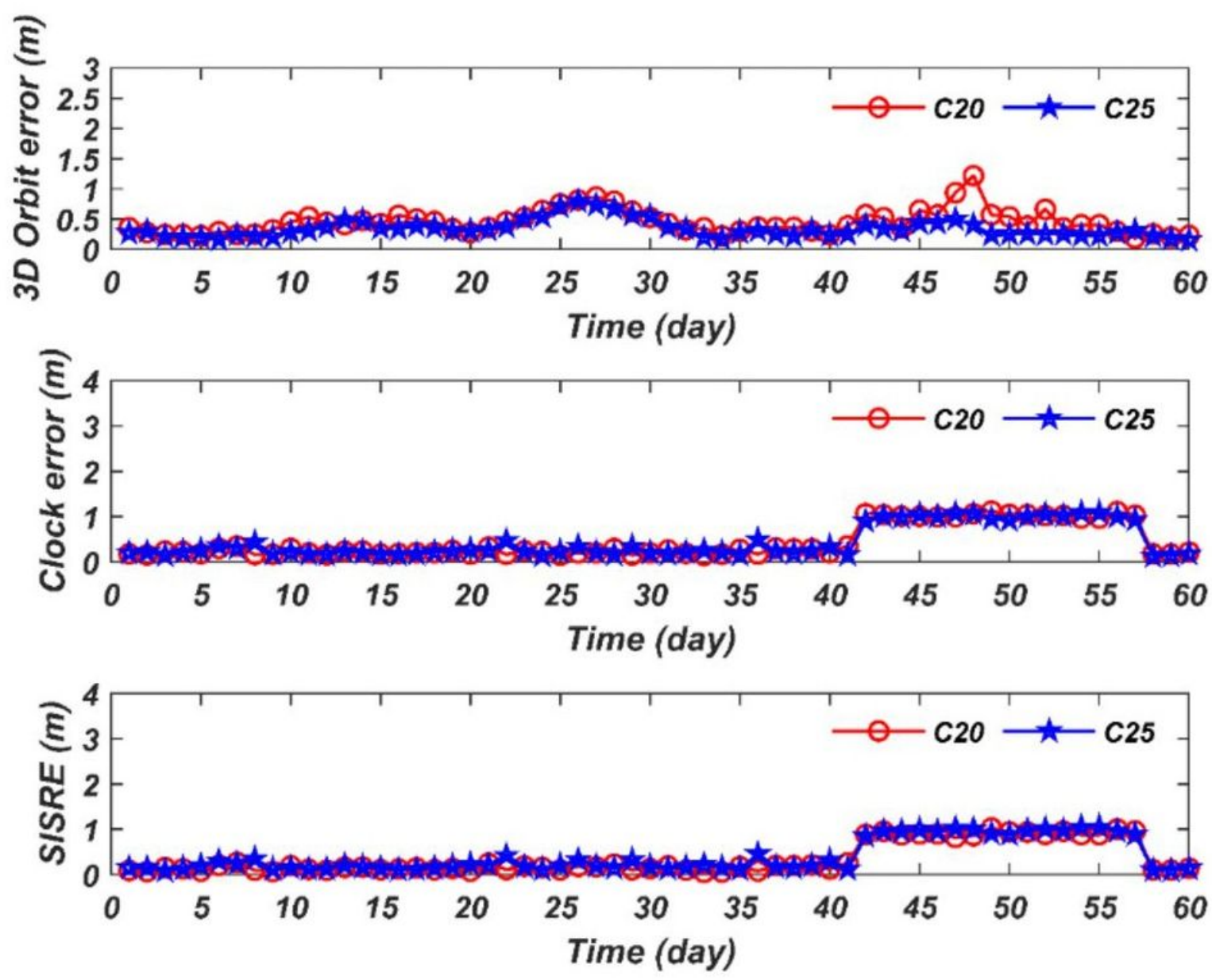

Figure 8

BDS-3 satellite 3D orbit error (RMS), Clock error (RMS) and SISRE (RMS)

(RMS)

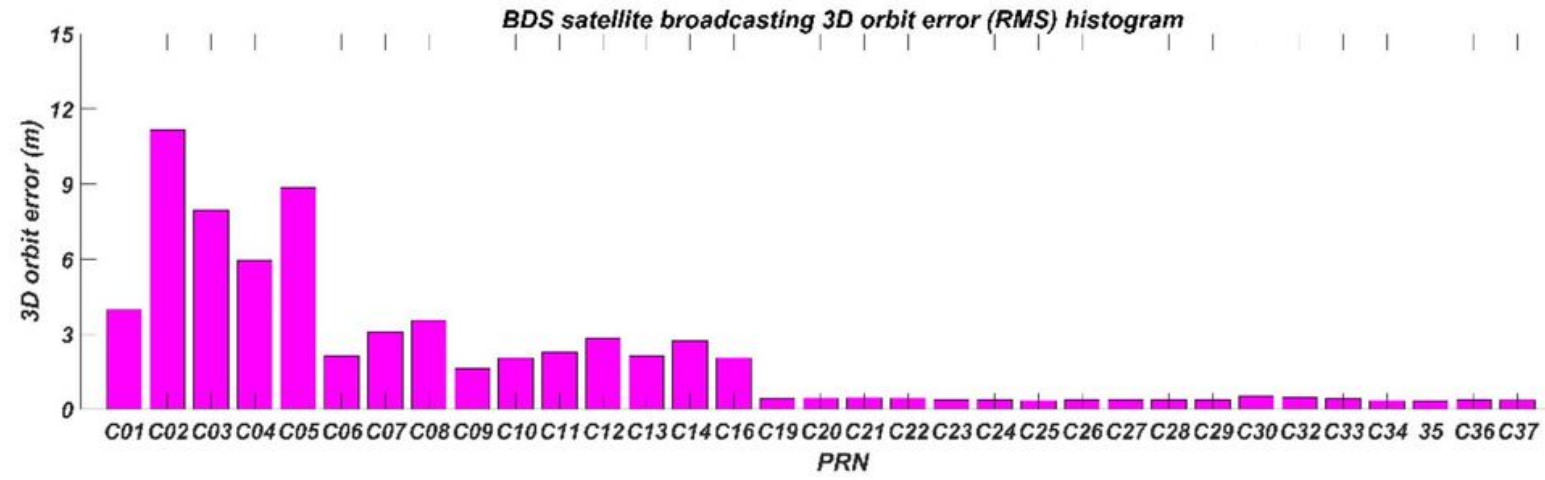

Figure 9 
BDS satellite broadcasting 3D orbit error (RMS) histogram

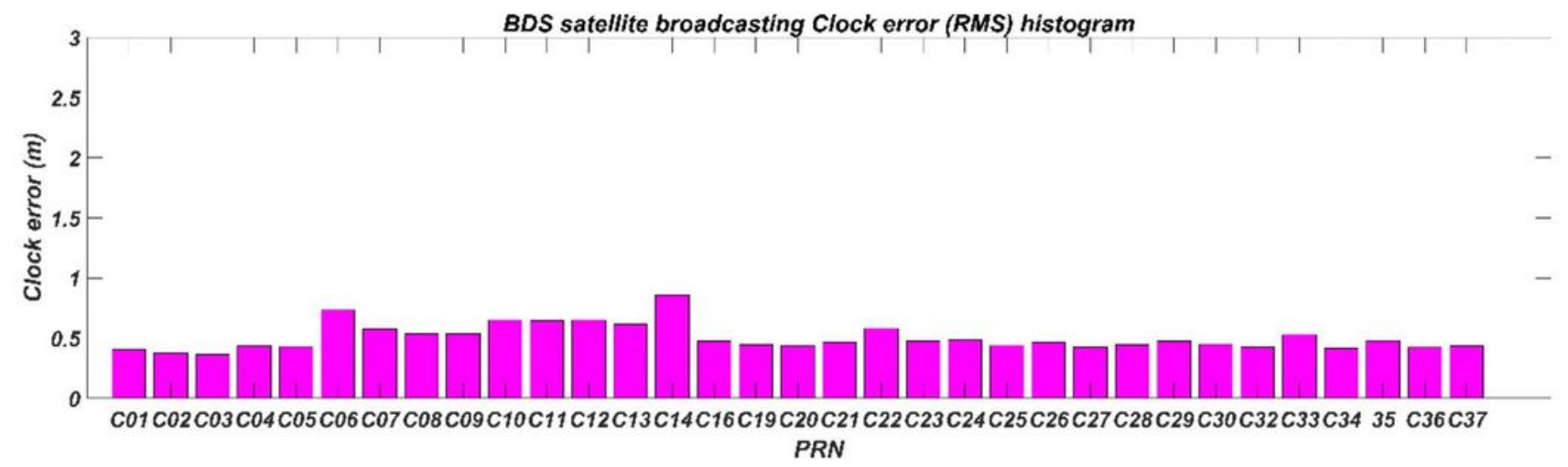

Figure 10

BDS satellite broadcasting Clock error (RMS) histogram

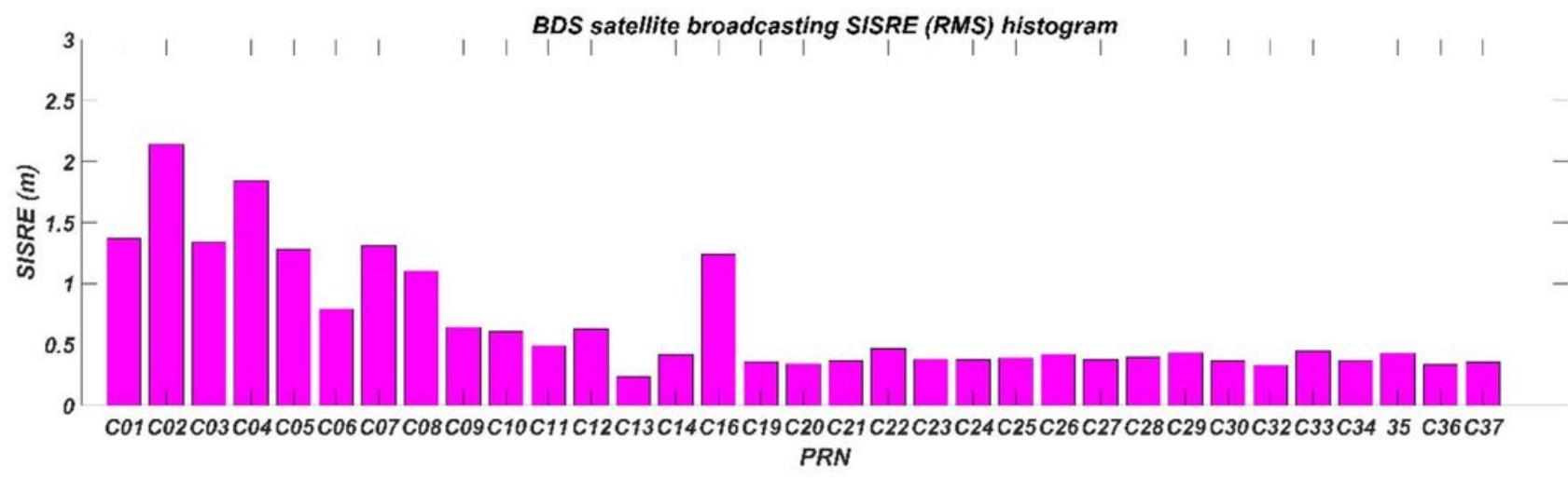

Figure 11

BDS satellite broadcasting SISRE (RMS) histogram

\section{Supplementary Files}

This is a list of supplementary files associated with this preprint. Click to download.

- GraphicalAbstract.png 